\title{
Synthesis, Characterization, and Cytotoxic Evaluation of Some Newly Substituted Diazene Candidates
}

\author{
Mohamed El-Naggar, ${ }^{1}$ Abdel-Nasser El-Shorbagi, ${ }^{2,3}$ Dina H. Elnaggar, ${ }^{4}$ \\ Abd El-Galil E. Amr $\mathbb{D}^{5,}{ }^{5,6}$ Mohamed A. Al-Omar, ${ }^{5}$ and Elsayed A. Elsayed $\mathbb{D}^{7,8}$ \\ ${ }^{1}$ Chemistry Department, Faculty of Sciences, University of Sharjah, Sharjah 27272, UAE \\ ${ }^{2}$ College of Pharmacy, University of Sharjah, Sharjah 27272, UAE \\ ${ }^{3}$ Sharjah Institute for Medical Research, University of Sharjah, Sharjah 27272, UAE \\ ${ }^{4}$ Applied Organic Chemistry Department, National Research Centre, Dokki, Giza 12622, Egypt \\ ${ }^{5}$ Drug Exploration \& Development Chair (DEDC), Pharmaceutical Chemistry Department, College of Pharmacy, \\ King Saud University, Riyadh 11451, Saudi Arabia \\ ${ }^{6}$ Department of Applied Organic Chemistry, Industrial Chemical Division, National Research Center, Cairo, Dokki 12622, Egypt \\ ${ }^{7}$ Bioproducts Research Department, Zoology Department, Faculty of Science, King Saud University, Riyadh, Saudi Arabia \\ ${ }^{8}$ Chemistry of Natural and Microbial Products Department, National Research Centre, Dokki 12622, Cairo, Egypt
}

Correspondence should be addressed to Abd El-Galil E. Amr; aeamr1963@yahoo.com

Received 18 June 2018; Revised 29 August 2018; Accepted 16 September 2018; Published 1 November 2018

Academic Editor: Artur M. S. Silva

Copyright (c) 2018 Mohamed El-Naggar et al. This is an open access article distributed under the Creative Commons Attribution License, which permits unrestricted use, distribution, and reproduction in any medium, provided the original work is properly cited.

\begin{abstract}
A series of azocompounds containing methyl salicylate $\mathbf{4 a - k}$ and 1-naphthyl moiety 6-8 was synthesized and tested as anticancer agents. Nitrosation of methyl 5-amino-2-hydroxybenzoate or 1-aminonaphthalene by using $\mathrm{NaNO}_{2}$ in the presence of $\mathrm{HCl}$ afforded diazonium salt derivatives $\mathbf{2}$ and $\mathbf{5}$, which were treated with substituted imino or substituted amino derivatives, to give the corresponding substituted amino-pent-2-en-3-yl-diazenylbenzoate $\mathbf{4 a - k}$ or 2-substituted-1-(naphthalen-1-yl)diazene derivatives $\mathbf{6 a}-\mathbf{h}, \mathbf{7 a}, \mathbf{b}$, and $\mathbf{8 a}, \mathbf{b}$. All the synthesized compounds were elucidated by elemental analysis and spectroscopic evidence.
\end{abstract}

\section{Introduction}

In a previous work, the authors reported that certain synthesized substituted heterocyclic aromatic derivatives showed antiviral activities [1] and alpha reductase inhibition [2]. Also, some of new compounds containing the nitrogen atom have been synthesized and used as antihypertensive $\alpha$-blocking [3], antiparkinsonian [4], antialzheimer [5], antimicrobial [6-8], and anti-inflammatory $[9,10]$ agents. There is an increasing and critical demand for more powerful anticancer agents, due to increased detection of cancer resistance [11]. Azocompounds are one of the largest types of organically synthesized compounds, which are effective both as drugs and cosmetics [12]. Also, they are extensively used in the field of dyeing textiles, biomedical studies, advanced applications in organic synthesis, and remarkable biological activities including antibacterial activity [13-20]. A number of azocompounds were synthesized comprising a drug moiety in their skeleton [21]. Salicylates are a class of chemicals with appreciative biological activities. Several biomedical studies have used methyl salicylate derivatives with promising results [22-24]. Aromatic amines such as benzidine, 1-naphthylamine, and 4-methylaniline have been recognized as potential carcinogens $[25,26]$. From these observations, we decided to synthesize two series of azocompounds. One of these series contains methyl salicylate moiety, and the other involves 1naphthyl moiety. All the synthesized compounds were screened as anticancer agents.

\section{Experimental}

2.1. Chemistry. All reagents and solvents were obtained from the commercial supplier and used without further purification. Melting point is uncorrected and was determined on an electrothermal melting point apparatus (Stuart Scientific, England, UK). Precoated silica gel plates 
(Kieselgel 0.25 mm, 60G $\mathrm{F}_{254}$, Merck, Germany) were used for thin layer chromatography (TLC) for reaction monitoring, and UV light was used for detection. The separation was done using column chromatography with silica gel 60 (Merck) eluted with appropriate solvent. Nuclear magnetic resonance spectra (NMR) were recorded using Bruker $500 \mathrm{MHz}$ instrument. Chemical shifts were reported in ppm, and spectra were corrected using the residual solvent as a reference. Mass spectra were recorded on a Bruker Daltonics spectrometer.

2.1.1. General Procedure for the Synthesis of Compounds 4a-k. A mixture of methyl 5-aminosalicylate 1 (0.56 g, $3.4 \mathrm{mmol}$ ) in concentrated hydrochloric acid $(5 \mathrm{~mL})$ was stirred with gentle heating until complete dissolving. Then, the mixture was cooled at a temperature from zero to $-4^{\circ} \mathrm{C}$, sodium nitrite $(0.28 \mathrm{~g}, 4 \mathrm{mmol})$ in $2 \mathrm{~mL}$ of water was added portionwise at a temperature of $-4^{\circ} \mathrm{C}$ to form the corresponding diazonium salt 2 , and the diazonium salt formed was added to a mixture of 4-(substituted imino)pentan-2-one derivatives 3 (2 mmol) [27] in ethanol $(15 \mathrm{~mL})$ slowly under the same temperature allowing the mixture to stir at room temperature for an additional 3 hours. The solid formed was collected by filtration, washed with cold water several times, and then left to dry. The obtained product was recrystallized from ethanol to give target compounds $4 \mathbf{a}-\mathbf{k}$, respectively.

(1) Methyl 5-(4-(benzylamino)-2-oxopent-3-en-3-yl) diazenyl)-2-hydroxybenzoate (4a). Yellow crystalline solid, m.p. $167-168^{\circ} \mathrm{C}$, yield $=62 \%$, and ${ }^{1} \mathrm{H}-\mathrm{NMR}\left(\mathrm{DMSO}^{\left.-\mathrm{d}_{6}\right)} \delta\right.$ (ppm) 2.42 (s, 3H, $\left.\mathrm{CH}_{3}-\mathrm{CNH}\right), 2.57$ (s, 3H, CO-CH $\left.{ }_{3}\right), 3.93$ $\left(\mathrm{s}, 3 \mathrm{H}, \mathrm{OCH}_{3}\right), 4.82\left(\mathrm{~s}, 2 \mathrm{H}, \mathrm{Ar}-\mathrm{CH}_{2}\right), 7.05(\mathrm{~d}, 1 \mathrm{H}, J=8.8 \mathrm{~Hz}$, Ar-H, benzoate ring), 7.36-7.42 (m, 5H, ArH), $7.72(\mathrm{~d}, 1 \mathrm{H}$, $J=2.5 \mathrm{~Hz}, \mathrm{Ar}-\mathrm{H}$, benzoate ring), $7.78(\mathrm{~s}, 1 \mathrm{H}, \mathrm{Ar}-\mathrm{H}$, benzoate ring), 10.50 (br s, $1 \mathrm{H}, \mathrm{NH}$ ), and $14.40(\mathrm{~s}, 1 \mathrm{H}, \mathrm{OH}) ;{ }^{13} \mathrm{C}-\mathrm{NMR}$ $\left(\mathrm{DMSO}_{\mathrm{d}}\right) \delta(\mathrm{ppm}) 16.8,29.0,47.6,53.0,113.8,118.9$, $123.0,125.6,128.1,128.2,128.6,129.4,137.3,144.5,159.1$, 159.4, 169.3, and 196.4 (20C). ESI-MS: $m / z, 368.16\left(\mathrm{M}^{+}+\mathrm{H}\right)$. Anal. calcd for $\mathrm{C}_{20} \mathrm{H}_{21} \mathrm{~N}_{3} \mathrm{O}_{4}$ (367.40): C, 65.38; $\mathrm{H}, 5.76$; and N, 11.44. Found: C, 65.35; H, 5.78; and N, 11.40 .

(2) Methyl 5-(2-oxo-4-(phenethylamino)pent-3-en-3-yl) diazenyl)-2-hydroxybenzoate (4b). Yellow crystalline solid, m.p. $131-132^{\circ} \mathrm{C}$, yield $=60 \%$, and ${ }^{1} \mathrm{H}-\mathrm{NMR}\left(\mathrm{DMSO}^{-\mathrm{d}_{6}}\right) \delta$ (ppm) 2.39 (s, 3H, CH $\left.\mathrm{CH}_{3} \mathrm{CNH}\right), 2.52$ (s, 3H, CO-CH $\left.{ }_{3}\right), 2.98$ $\left(\mathrm{t}, 2 \mathrm{H}, J=6.8 \mathrm{~Hz}, \mathrm{PhCH}_{2} \mathrm{CH}_{2} \mathrm{~N}\right), 3.85(\mathrm{t}, 2 \mathrm{H}, J=6.7 \mathrm{~Hz}$, $\mathrm{ArCH}_{2} \mathrm{CH}_{2} \mathrm{~N}$ ), 3.95 (s, 3H, $\left.\mathrm{OCH}_{3}\right), 7.03$ (d, $1 \mathrm{H}, J=8.8 \mathrm{~Hz}$, Ar- $\mathrm{H}$, benzoate ring), 7.34-7.30 (m, 5H, ArH), 7.59 (d, 1H, J $=2.5 \mathrm{~Hz}, \mathrm{Ar}-\mathrm{H}$, benzoate ring), $7.76(\mathrm{~s}, 1 \mathrm{H}, \mathrm{Ar}-\mathrm{H}$, benzoate ring), 10.50 (br s, $1 \mathrm{H}, \mathrm{NH})$, and $13.84(\mathrm{~s}, 1 \mathrm{H}, \mathrm{OH}) ;{ }^{13} \mathrm{C}-\mathrm{NMR}$ $\left(\right.$ DMSO-d $\left._{6}\right) \delta$ (ppm) 16.3, 29.1, 35.0, 44.6, 53.1, 113.8, 118.7, $122.4,126.6,127.0,128.3,129.0,129.2,138.7,144.9,159.1$, 159.3, 169.5, and 196.3 (21C). ESI-MS: $m / z, 382.18\left(\mathrm{M}^{+}+\mathrm{H}\right)$. Anal. calcd for $\mathrm{C}_{21} \mathrm{H}_{23} \mathrm{~N}_{3} \mathrm{O}_{4}$ (381.43): C, 66.13; $\mathrm{H}, 6.08$; and N, 11.02. Found: C, 66.45; H, 6.02; and N, 11.00 .

(3) Methyl 5-(2-oxo-4-(1-phenylethylamino)pent-3-en-3yl)diazenyl)-2-hydroxybenzoate (4c). Yellow crystalline solid, m.p. $110-111^{\circ} \mathrm{C}$, yield $=66 \%$, and ${ }^{1} \mathrm{H}-\mathrm{NMR}$ (DMSO$\left.\mathrm{d}_{6}\right) \delta(\mathrm{ppm}) 1.60\left(\mathrm{~d}, 3 \mathrm{H}, J=7.6 \mathrm{~Hz}, \operatorname{ArCH}\left(\mathrm{CH}_{3}\right) \mathrm{N}\right), 2.41$ (s, $\left.3 \mathrm{H}, \mathrm{CH}_{3}-\mathrm{C}\right), 2.49$ (s, 3H, CO- $\mathrm{CH}_{3}$ ), 3.94 (s, $3 \mathrm{H}, \mathrm{OCH}_{3}$ ), $5.17\left(\mathrm{q}, 1 \mathrm{H}, J=6.7 \mathrm{~Hz}, \operatorname{ArCH}\left(\mathrm{CH}_{3}\right) \mathrm{N}\right), 7.06(\mathrm{~d}, 1 \mathrm{H}, J=$ 8.8 Hz, Ar-H, benzoate ring), 7.40-7.45 (m, 5H, ArH), 7.76 (d, $1 \mathrm{H}, J=2.5 \mathrm{~Hz}, \mathrm{Ar}-\mathrm{H}$, benzoate ring), 7.86 (s, $1 \mathrm{H}, \mathrm{Ar}-\mathrm{H}$, benzoate ring), 10.50 (br s, $1 \mathrm{H}, \mathrm{NH}$ ), and 14.63 (s, 1H, OH); ${ }^{13}$ C-NMR (DMSO-d $\mathrm{d}_{6}$ ) $\delta$ (ppm) 16.8, 24.0, 29.0, 53.1, 53.7, $114.0,119.0,122.2,126.4,126.6,128.2,128.5,129.4,143.0$, $144.5,159.2,159.4,169.3$, and $196.4(21 \mathrm{C})$. ESI-MS: $m / z$, $382.18\left(\mathrm{M}^{+}+\mathrm{H}\right)$. Anal. calcd for $\mathrm{C}_{21} \mathrm{H}_{23} \mathrm{~N}_{3} \mathrm{O}_{4}$ (381.43): $\mathrm{C}$, 66.13; H, 6.08; and N, 11.02. Found: C, 66.04; H, 6.03; and N, 10.98 .

(4) Methyl 5-(4-(2-hydroxyethylamino)-2-oxopent-3-en3-yl)diazenyl)-2-hydroxybenzoate (4d). Yellow crystalline solid, m.p. $187-188^{\circ} \mathrm{C}$, yield $=70 \%$, and ${ }^{1} \mathrm{H}-\mathrm{NMR}$ (DMSO$\left.\mathrm{d}_{6}\right) \delta(\mathrm{ppm}) 2.42\left(\mathrm{~s}, 3 \mathrm{H}, \mathrm{CH}_{3}-\mathrm{C}\right), 2.54\left(\mathrm{~s}, 3 \mathrm{H}, \mathrm{CO}-\mathrm{CH}_{3}\right)$, $3.59\left(\mathrm{t}, 2 \mathrm{H}, J=5.0 \mathrm{~Hz}, \mathrm{NCH}_{2} \mathrm{CH}_{2} \mathrm{OH}\right), 3.66(\mathrm{t}, 2 \mathrm{H}, J=$ $4.2 \mathrm{~Hz}, \mathrm{NCH}_{2} \mathrm{CH}_{2} \mathrm{OH}$ ), $3.92\left(\mathrm{~s}, 3 \mathrm{H}, \mathrm{OCH}_{3}\right), 5.13$ (br s, $1 \mathrm{H}$, $\left.\mathrm{NCH}_{2} \mathrm{CH}_{2} \mathrm{OH}\right) 7.07$ (d, $1 \mathrm{H}, J=8.8 \mathrm{~Hz}, \mathrm{Ar}-\mathrm{H}$, benzoate ring), $7.79(\mathrm{~d}, 1 \mathrm{H}, J=2.5 \mathrm{~Hz}$, Ar- $\mathrm{H}$, benzoate ring), $8.01(\mathrm{~s}, 1 \mathrm{H}$, Ar- $\mathrm{H}$, benzoate ring), and 10.5 (br s, $1 \mathrm{H}, \mathrm{NH}), 14.21$ (s, $1 \mathrm{H}$, $\mathrm{OH}) ;{ }^{13} \mathrm{C}-\mathrm{NMR}$ (DMSO-d 6 ) $\delta$ (ppm) 16.7, 29.1, 46.0, 53.1, 59.7, 113.9, 118.7, 122.1, 127.0, 128.4, 134.5, 154.1, 159.3, 169.5, and 196.4 (15C). ESI-MS: $322.27\left(\mathrm{M}^{+}+\mathrm{H}\right)$. Anal. calcd for $\mathrm{C}_{15} \mathrm{H}_{19} \mathrm{~N}_{3} \mathrm{O}_{5}$ (321.33): C, 56.07; $\mathrm{H}, 5.96$; and $\mathrm{N}$, 13.08. Found: C, 56.00; H, 5.92; and N, 13.00 .

(5) Methyl 5-(4-(isobutylamino)-2-oxopent-3-en-3-yl) diazenyl)-2-hydroxybenzoate (4e). Yellow crystalline solid, m.p. $94-95^{\circ} \mathrm{C}$, yield $=75 \%$, and ${ }^{1} \mathrm{H}-\mathrm{NMR}\left(\mathrm{DMSO}-\mathrm{d}_{6}\right)$ $\delta(\mathrm{ppm}) 1.00\left(\mathrm{~d}, 6 \mathrm{H}, J=6.7 \mathrm{~Hz}, \mathrm{NHCH}_{2} \mathrm{CH}\left(\mathrm{CH}_{3}\right)_{2}\right), 1.95-$ $1.90\left(\mathrm{~m}, 1 \mathrm{H}, \mathrm{NHCH}_{2} \mathrm{CH}\left(\mathrm{CH}_{3}\right)_{2}\right), 2.42$ (s, 3H, $\left.\mathrm{CH}_{3}-\mathrm{C}\right), 2.54$ (s, $\left.3 \mathrm{H}, \mathrm{CO}-\mathrm{CH}_{3}\right), 3.39\left(\mathrm{dd}, 2 \mathrm{H}, J=5.4 \mathrm{~Hz}, \mathrm{NHCH}_{2} \mathrm{CH}\left(\mathrm{CH}_{3}\right)_{2}\right)$, $3.92\left(\mathrm{~s}, 3 \mathrm{H}, \mathrm{OCH}_{3}\right), 7.07$ (d, $1 \mathrm{H}, J=8.8 \mathrm{~Hz}, \mathrm{Ar}-\mathrm{H}$, benzoate ring), $7.76(\mathrm{~d}, 1 \mathrm{H}, J=2.5 \mathrm{~Hz}$, Ar-H, benzoate ring), 7.95 (s, 1H, Ar-H, benzoate ring), 10.50 (br s, 1H, NH), and 14.09 (s, $1 \mathrm{H}, \mathrm{OH}) ;{ }^{13} \mathrm{C}-\mathrm{NMR}$ (DMSO-d 6 ) $\delta(\mathrm{ppm}) 16.45,2.36,28.3$, 29.1, 50.7, 53.1, 114.0, 118.9, 122.0, 126.8, 128.4, 134.5, 145.2, 159.3, 169.4, and 196.2 (17C). ESI-MS: $m / z, 334.18\left(\mathrm{M}^{+}+\mathrm{H}\right)$. Anal. calcd for $\mathrm{C}_{17} \mathrm{H}_{23} \mathrm{~N}_{3} \mathrm{O}_{4}$ (333.38): C, 61.25; $\mathrm{H}, 6.95$; and $\mathrm{N}$, 12.60. Found: C, 61.12; H, 6.90; and N, 12.54 .

(6) Methyl 5-(4-(isopropylamino)-2-oxopent-3-en-3-yl) diazenyl)-2-hydroxybenzoate (4f). Yellow crystalline solid, m.p. $104-105^{\circ} \mathrm{C}$, yield $=61 \%$, and ${ }^{1} \mathrm{H}-\mathrm{NMR}\left(\mathrm{DMSO}-\mathrm{d}_{6}\right)$ $\delta(\mathrm{ppm}) 1.30\left(\mathrm{~d}, 6 \mathrm{H}, J=6.4 \mathrm{~Hz}, \mathrm{NHCH}\left(\mathrm{CH}_{3}\right)_{2}\right), 2.41(\mathrm{~s}, 3 \mathrm{H}$, $\left.\mathrm{CH}_{3}-\mathrm{C}\right), 2.56$ (s, 3H, $\left.\mathrm{COCH}_{3}\right), 3.92$ (s, 3H, $\left.\mathrm{OCH}_{3}\right), 4.15-4.12$ $\left(\mathrm{m}, 1 \mathrm{H}, J=6.4 \mathrm{~Hz}, \mathrm{NHCH}\left(\mathrm{CH}_{3}\right)_{2}\right), 7.08(\mathrm{~d}, 1 \mathrm{H}, J=8.8 \mathrm{~Hz}$, Ar- $\mathrm{H}$, benzoate ring), $7.78(\mathrm{~d}, 1 \mathrm{H}, J=2.5 \mathrm{~Hz}, \mathrm{Ar}-\mathrm{H}$, benzoate ring), 7.92 (s, 1H, Ar-H, benzoate ring), 10.50 (br s, $1 \mathrm{H}, \mathrm{NH}$ ), and $14.10(\mathrm{~s}, 1 \mathrm{H}, \mathrm{OH}) ;{ }^{13} \mathrm{C}-\mathrm{NMR}\left(\mathrm{DMSO}_{-} \mathrm{d}_{6}\right) \delta(\mathrm{ppm}) 16.3$, 23.2, 29.1, 45.6, 53.1, 114.0, 118.8, 121.8, 127.1, 127.9, 134.5, $145.1,159.3,169.4$, and 196.1 (16C). ESI-MS: $m / z, 320.16$ $\left(\mathrm{M}^{+}+\mathrm{H}\right)$. Anal. calcd for $\mathrm{C}_{16} \mathrm{H}_{21} \mathrm{~N}_{3} \mathrm{O}_{4}$ (319.36): C, 60.17; $\mathrm{H}$, 6.63; and N, 13.16. Found: C, 60.10; H, 6.58; and N, 13.10.

(7) Methyl 5-(4-(butylamino)-2-oxopent-3-en-3-yl) diazenyl)-2-hydroxybenzoate (4g). Yellow crystalline solid, m.p. $131-132^{\circ} \mathrm{C}$, yield $=65 \%$, and ${ }^{1} \mathrm{H}-\mathrm{NMR}\left(\mathrm{DMSO}-\mathrm{d}_{6}\right)$ $\delta(\mathrm{ppm}) 0.97\left(\mathrm{t}, 3 \mathrm{H}, J=7.3 \mathrm{~Hz}, \mathrm{NHCH}_{2} \mathrm{CH}_{2} \mathrm{CH}_{2} \mathrm{CH}_{3}\right), 1.43-$ $1.40\left(\mathrm{~m}, 2 \mathrm{H}, J=7.6 \mathrm{~Hz}, \mathrm{NHCH}_{2} \mathrm{CH}_{2} \mathrm{CH}_{2} \mathrm{CH}_{3}\right), 1.62-1.66$ $\left(\mathrm{m}, 2 \mathrm{H}, J=6.9 \mathrm{~Hz}, \mathrm{NHCH}_{2} \mathrm{CH}_{2} \mathrm{CH}_{2} \mathrm{CH}_{3}\right), 2.41(\mathrm{~s}, 3 \mathrm{H}$, $\left.\mathrm{CH}_{3}-\mathrm{C}\right), 2.53\left(\mathrm{~s}, 3 \mathrm{H}, \mathrm{COCH}_{3}\right), 3.52(\mathrm{t}, 2 \mathrm{H}, J=6.7 \mathrm{~Hz}$, $\left.\mathrm{NHCH}_{2} \mathrm{CH}_{2} \mathrm{CH}_{2} \mathrm{CH}_{3}\right), 3.91\left(\mathrm{~s}, 3 \mathrm{H}, \mathrm{OCH}_{3}\right), 7.07$ (d, $1 \mathrm{H}$, 
$J=8.8 \mathrm{~Hz}, \mathrm{Ar}-\mathrm{H}$, benzoate ring), $7.72(\mathrm{~d}, 1 \mathrm{H}, J=2.5 \mathrm{~Hz}, \mathrm{Ar}-$ $\mathrm{H}$, benzoate ring), $7.93(\mathrm{~s}, 1 \mathrm{H}, \mathrm{Ar}-\mathrm{H}$, benzoate ring), 10.50 (br s, $1 \mathrm{H}, \mathrm{NH}$ ), and $14.05(\mathrm{~s}, 1 \mathrm{H}, \mathrm{OH}) ;{ }^{13} \mathrm{C}$-NMR (DMSO$\left.\mathrm{d}_{6}\right) \delta(\mathrm{ppm}) 14.0,16.4,20.2,29.0,31.0,43.1,53.1,113.9$, $118.9,122.6,126.5,128.2,134.5,145.1,159.3,169.4$, and 196.2 (17C). ESI-MS: $m / z, 334.18\left(\mathrm{M}^{+}+\mathrm{H}\right)$. Anal. calcd for $\mathrm{C}_{17} \mathrm{H}_{23} \mathrm{~N}_{3} \mathrm{O}_{4}$ (333.38): C, 61.25; $\mathrm{H}, 6.95$; and $\mathrm{N}, 12.60$. Found: C, 61.12; H, 6.86; and N, 12.52 .

(8) Methyl 5-(4-(3-hydroxypropylamino)-2-oxopent-3en-3-yl)diazenyl)-2-hydroxybenzoate (4h). Yellow crystalline solid, m.p. $135-136^{\circ} \mathrm{C}$, yield $=63 \%$, and ${ }^{1} \mathrm{H}-\mathrm{NMR}\left(\mathrm{DMSO}-\mathrm{d}_{6}\right)$ $\delta(\mathrm{ppm}) 1.80-1.75\left(\mathrm{~m}, 2 \mathrm{H}, J=6.3 \mathrm{~Hz}, \mathrm{NHCH}_{2} \mathrm{CH}_{2} \mathrm{CH}_{2} \mathrm{OH}\right)$, $2.41\left(\mathrm{~s}, 3 \mathrm{H}, \mathrm{CH}_{3}-\mathrm{C}\right), 2.54\left(\mathrm{~s}, 3 \mathrm{H}, \mathrm{CO}-\mathrm{CH}_{3}\right), 3.54$ (br t, $2 \mathrm{H}, J=$ $5.7 \mathrm{~Hz}, \mathrm{NHCH}_{2} \mathrm{CH}_{2} \mathrm{CH}_{2} \mathrm{OH}$ ), 3.59 (br t, $2 \mathrm{H}, J=6.7 \mathrm{~Hz}$, $\mathrm{NHCH}_{2} \mathrm{CH}_{2} \mathrm{CH}_{2} \mathrm{OH}$ ), 3.91 (s, $3 \mathrm{H}, \mathrm{OCH}_{3}$ ), 4.70 (br s, $2 \mathrm{H}$, $\left.\mathrm{NHCH}_{2} \mathrm{CH}_{2} \mathrm{CH}_{2} \mathrm{OH}\right), 7.07$ (d, $1 \mathrm{H}, J=8.8 \mathrm{~Hz}, \mathrm{Ar}-\mathrm{H}$, benzoate ring), $7.77(\mathrm{~d}, 1 \mathrm{H}, J=2.5 \mathrm{~Hz}, \mathrm{Ar}-\mathrm{H}$, benzoate ring), $7.98(\mathrm{~s}, 1 \mathrm{H}$, Ar- $\mathrm{H}$, benzoate ring), and 10.50 (br s, $1 \mathrm{H}, \mathrm{NH}), 13.95(\mathrm{~s}, 1 \mathrm{H}$, $\mathrm{OH}$ ); ${ }^{13} \mathrm{C}$-NMR (DMSO-d ${ }_{6}$ ) $\delta$ (ppm) 16.3, 29.1, 32.2, 40.0, 53.1, 58.4, 113.9, 118.7, 122.0, 127.1, 128.3, 134.5, 145.2, 159.3, 169.5, and 196.2 (16C). ESI-MS): $m / z, 336.16\left(\mathrm{M}^{+}+\mathrm{H}\right)$. Anal. calcd for $\mathrm{C}_{16} \mathrm{H}_{21} \mathrm{~N}_{3} \mathrm{O}_{5}$ (335.36): C, 57.30; H, 6.31; and N, 12.53. Found: C, 57.10; $\mathrm{H}, 6.22$; and $\mathrm{N}, 12.42$.

(9) Methyl 5-(2-oxo-4-(pentylamino)pent-3-en-3-yl) diazenyl)-2-hydroxybenzoate (4i). Yellow crystalline solid, m.p. $127-128^{\circ} \mathrm{C}$, yield $=72 \%$, and ${ }^{1} \mathrm{H}-\mathrm{NMR}\left(\mathrm{DMSO}^{-\mathrm{d}_{6}}\right)$ $\delta(\mathrm{ppm}) 0.92\left(\mathrm{t}, 3 \mathrm{H}, J=6.9 \mathrm{~Hz}, \mathrm{NHCH}_{2} \mathrm{CH}_{2} \mathrm{CH}_{2} \mathrm{CH}_{2} \mathrm{CH}_{3}\right)$, 1.40-1.45 (m, $\left.4 \mathrm{H}, \quad \mathrm{NHCH}_{2} \mathrm{CH}_{2} \mathrm{CH}_{2} \mathrm{CH}_{2} \mathrm{CH}_{3}\right), 1.66-1.62$ (m, $\left.2 \mathrm{H}, \mathrm{NHCH}_{2} \mathrm{CH}_{2} \mathrm{CH}_{2} \mathrm{CH}_{2} \mathrm{CH}_{3}\right), 2.41$ (s, 3H, $\mathrm{CH}_{3}-\mathrm{C}$ ), $2.54\left(\mathrm{~s}, \quad 3 \mathrm{H}, \quad \mathrm{CO}-\mathrm{CH}_{3}\right), \quad 3.53(\mathrm{t}, 2 \mathrm{H}, \quad J=6.7 \mathrm{~Hz}$, $\left.\mathrm{NHCH}_{2} \mathrm{CH}_{2} \mathrm{CH}_{2} \mathrm{CH}_{2} \mathrm{CH}_{3}\right), 3.91\left(\mathrm{~s}, 3 \mathrm{H}, \mathrm{OCH}_{3}\right), 7.06$ (d, $1 \mathrm{H}$, $J=8.8 \mathrm{~Hz}$, Ar- $\mathrm{H}$, benzoate ring), $7.78(\mathrm{~d}, 1 \mathrm{H}, J=2.5, \mathrm{Ar}-\mathrm{H}$, benzoate ring), $7.93(\mathrm{~s}, 1 \mathrm{H}, \mathrm{Ar}-\mathrm{H}$, benzoate ring), 10.50 (br s, $1 \mathrm{H}, \mathrm{NH}$ ), and $14.05(\mathrm{~s}, 1 \mathrm{H}, \mathrm{OH}) ;{ }^{13} \mathrm{C}-\mathrm{NMR}\left(\mathrm{DMSO}-\mathrm{d}_{6}\right)$ $\delta(\mathrm{ppm}) 14.3,16.4,22.3,28.6,29.2,30.7,43.4,53.0,113.8$, $118.9,122.7,126.3,128.2,134.5,145.1,159.4,169.4$, and 196.2 (18C). ESI-MS: $m / z, 348.19\left(\mathrm{M}^{+}+\mathrm{H}\right)$. Anal. calcd for $\mathrm{C}_{18} \mathrm{H}_{25} \mathrm{~N}_{3} \mathrm{O}_{4}$ (347.41): $\mathrm{C}, 62.23 ; \mathrm{H}, 7.25$; and N, 12.10. Found: $\mathrm{C}, 62.05 ; \mathrm{H}, 7.16$; and $\mathrm{N}, 12.05$.

(10) Methyl 5-(4-(ethylamino)-2-oxopent-3-en-3-yl) diazenyl)-2-hydroxybenzoate (4j). Yellow crystalline solid, m.p. $106-107^{\circ} \mathrm{C}$, yield $=75 \%$, and ${ }^{1} \mathrm{H}-\mathrm{NMR}$ (DMSO$\left.\mathrm{d}_{6}\right) \delta(\mathrm{ppm}) 1.27\left(\mathrm{t}, 3 \mathrm{H}, J=7.2 \mathrm{~Hz}, \mathrm{NHCH}_{2} \mathrm{CH}_{3}\right), 2.41(\mathrm{~s}, 3 \mathrm{H}$, $\left.\mathrm{CH}_{3}-\mathrm{C}\right), 2.54$ (s, $\left.3 \mathrm{H}, \mathrm{CO}-\mathrm{CH}_{3}\right), 3.55$ (q, $2 \mathrm{H}, \mathrm{NHCH}_{2} \mathrm{CH}_{3}$ ), $3.91\left(\mathrm{~s}, 3 \mathrm{H}, \mathrm{OCH}_{3}\right), 7.07(\mathrm{~d}, 1 \mathrm{H}, J=8.8 \mathrm{~Hz}, \mathrm{Ar}-\mathrm{H}$, benzoate ring), $7.78(\mathrm{~d}, 1 \mathrm{H}, J=2.5 \mathrm{~Hz}, \mathrm{Ar}-\mathrm{H}$, benzoate ring), 7.93 (s, $1 \mathrm{H}, \mathrm{Ar}-\mathrm{H}$, benzoate ring), 10.50 (br s, $1 \mathrm{H}, \mathrm{NH}$ ), and 13.88 (s, $1 \mathrm{H}, \mathrm{OH}) ;{ }^{13} \mathrm{C}-\mathrm{NMR}$ (DMSO-d 6 ) $\delta$ (ppm) 14.8, 16.3, 29.1, $38.3,53.1,113.9,118.8,122.3,126.9,128.1,134.5,145.3$, 159.4, 169.5, and 196.1 (15C). ESI-MS): $m / z, 306.15\left(\mathrm{M}^{+}+\right.$ $\mathrm{H})$. Anal. calcd for $\mathrm{C}_{15} \mathrm{H}_{19} \mathrm{~N}_{3} \mathrm{O}_{4}$ (305.33): C, 59.01; $\mathrm{H}, 6.27$; and N, 13.76. Found: C, 58.86; H, 6.20; and N, 13.70.

(11) Methyl 5-(2-oxo-4-(propylamino)pent-3-en-3-yl) diazenyl)-2-hydroxybenzoate (4k). Yellow crystalline solid, m.p. $137-138^{\circ} \mathrm{C}$, yield $=69 \%$, and ${ }^{1} \mathrm{H}-\mathrm{NMR}\left(\mathrm{DMSO}_{-} \mathrm{d}_{6}\right) \delta$ (ppm) $0.99\left(\mathrm{t}, 3 \mathrm{H}, J=7.3 \mathrm{~Hz}, \mathrm{NHCH}_{2} \mathrm{CH}_{2} \mathrm{CH}_{3}\right), 1.68-1.64$ $\left(\mathrm{m}, 3 \mathrm{H}, J=7.2 \mathrm{~Hz}, \mathrm{NHCH}_{2} \mathrm{CH}_{2} \mathrm{CH}_{3}\right), 2.41\left(\mathrm{~s}, 3 \mathrm{H}, \mathrm{CH}_{3}-\mathrm{C}\right)$, $2.54\left(\mathrm{~s}, 3 \mathrm{H}, \mathrm{CO}-\mathrm{CH}_{3}\right), 3.50\left(\mathrm{q}, 2 \mathrm{H}, J=6.9 \mathrm{~Hz}, \mathrm{NHCH}_{2} \mathrm{CH}_{3}\right)$, $3.92\left(\mathrm{~s}, 3 \mathrm{H}, \mathrm{OCH}_{3}\right), 7.08(\mathrm{~d}, 1 \mathrm{H}, J=8.8 \mathrm{~Hz}, \mathrm{Ar}-\mathrm{H}$, benzoate ring), 7.77 (d, $1 \mathrm{H}, J=2.5 \mathrm{~Hz}, \mathrm{Ar}-\mathrm{H}$, benzoate ring), 7.96 (s, $1 \mathrm{H}, \mathrm{Ar}-\mathrm{H}$, benzoate ring), 10.52 (br s, $1 \mathrm{H}, \mathrm{NH}$ ), and 13.98 (s, $1 \mathrm{H}, \mathrm{OH}) ;{ }^{13} \mathrm{C}-\mathrm{NMR}\left(\mathrm{DMSO}_{\mathrm{d}}\right) \delta(\mathrm{ppm}) 11.8,16.4,22.5$, 29.1, 45.1, 53.1, 114, 118.8, 122.2, 126.9, 128.3, 134.5, 145.2, 159.3, 169.4, and 196.2 (16C). ESI-MS: $m / z, 320.14\left(\mathrm{M}^{+}+\mathrm{H}\right)$. Anal. calcd for $\mathrm{C}_{16} \mathrm{H}_{21} \mathrm{~N}_{3} \mathrm{O}_{4}$ (319.36): $\mathrm{C}, 60.17 ; \mathrm{H}, 6.63$; and $\mathrm{N}, 13.16$. Found: C, 60.00; H, 6.56; and N, 13.10.

2.1.2. General Procedure for the Synthesis of Compounds 6-8. A mixture of 1-aminonaphthalene $(0.48 \mathrm{~g}, 3.4 \mathrm{mmol})$ and conc. $\mathrm{HCl}(5 \mathrm{~mL})$ was stirred with gentle heating until complete dissolving. The reaction mixture was cooled at a temperature from zero to $-4^{\circ} \mathrm{C}$ in an ice bath, and then sodium nitrite $(0.28 \mathrm{~g}, 4 \mathrm{mmol})$ in water $(2 \mathrm{~mL})$ was added portionwise to the reaction mixture at the same temperature to form the diazonium salt 5 . To the diazonium salt 5 , a solution of appropriate amide or iminoderivatives [27], namely, $\mathrm{N}$-substituted 3-oxo-butanamide, ethyl 3-(substituted imino) butanoate, or $\mathrm{N}$-alkyl-3-(alkyl imino)-butanamide in absolute ethanol $(10 \mathrm{~mL})$, was added while stirring at a temperature of $-4^{\circ} \mathrm{C}$, and the mixture was stirred at room temperature for an additional 3 hours. The solid formed was collected by filtration, washed with cold water several times, dried, and crystallized from dimethylformamide/ethanol to give the corresponding $\mathbf{6 a}-\mathbf{h}, 7 \mathbf{a}, \mathbf{b}$, and $\mathbf{8 a}, \mathbf{b}$, respectively.

(1) 2-(Naphthalen-1-yl-diazenyl)-3-hydroxy-N-phenethylbut2-enamide (6a). Brownish crystalline solid, m.p. $141-142^{\circ} \mathrm{C}$, yield $=70 \%$, and ${ }^{1} \mathrm{H}-\mathrm{NMR}\left(\mathrm{DMSO}-\mathrm{d}_{6}\right) \delta(\mathrm{ppm}) 2.50(\mathrm{~s}, 3 \mathrm{H}$, C- $\left.\mathrm{CH}_{3}\right), 2.88\left(\mathrm{t}, 2 \mathrm{H}, J=7.2 \mathrm{~Hz}, \mathrm{ArCH}_{2} \mathrm{CH}_{2} \mathrm{NH}\right), 3.62(\mathrm{q}, 2 \mathrm{H}$, $\left.J=7.0 \mathrm{~Hz}, \mathrm{ArCH}_{2} \mathrm{CH}_{2} \mathrm{NH}\right), 7.23-7.20(\mathrm{~m}, 1 \mathrm{H}, \mathrm{Ar}), 7.33-7.30$ (m, 4H, ArH), 7.63-7.60 (m, 2H, naphthyl-H), 7.74-7.70 (m, $1 \mathrm{H}$, naphthyl-H), $7.78(\mathrm{~d}, 1 \mathrm{H}, J=8.1 \mathrm{~Hz}$, naphthyl- $\mathrm{H}), 7.83$ $(\mathrm{d}, 1 \mathrm{H}, J=6.7 \mathrm{~Hz}$, naphthyl-H), $7.91(\mathrm{~d}, 1 \mathrm{H}, J=8.4 \mathrm{~Hz}$, naphthyl-H), 8.04 (d, $1 \mathrm{H}, J=7.9 \mathrm{~Hz}$, naphthyl-H), 9.40 (br s, $1 \mathrm{H}, \mathrm{NHCO})$, and $15.67(\mathrm{~s}, 1 \mathrm{H}, \mathrm{OH}) ;{ }^{13} \mathrm{C}-\mathrm{NMR}$ (DMSO-d $\left.\mathrm{d}_{6}\right)$ $\delta(\mathrm{ppm}) 26.4,35.2,40.7,110.9,119.8,123.1,125.1,126.8$, $126.9,127.2,127.5,128.1,128.9,129.2,129.3,134.2,136.9$, 139.5, 164.9, and 198.7 (22C). ESI- MS: $m / z, 360.17\left(\mathrm{M}^{+}+\right.$ H). Anal. calcd for $\mathrm{C}_{22} \mathrm{H}_{21} \mathrm{~N}_{3} \mathrm{O}_{2}$ (359.42): C, 73.52; $\mathrm{H}, 5.89$; and N, 11.69. Found: C, 73.45; H, 5.80; and N, 11.60.

(2) 2-(Naphthalen-1-yl-diazenyl)-3-hydroxy-N-methylbut2-enamide (6b). Brownish crystalline solid, m.p. $175-176^{\circ} \mathrm{C}$, yield $=71 \%$, and ${ }^{1} \mathrm{H}-\mathrm{NMR}\left(\mathrm{DMSO}_{-} \mathrm{d}_{6}\right) \delta(\mathrm{ppm}) 2.52(\mathrm{~s}, 3 \mathrm{H}$, C- $\left.\mathrm{CH}_{3}\right), 2.88\left(\mathrm{~d}, 3 \mathrm{H}, J=4.9 \mathrm{~Hz}, \mathrm{CONHCH}_{3}\right), 7.63-7.60$ (m, $2 \mathrm{H}$, naphthyl-H), 7.72-7.68 $(\mathrm{m}, 1 \mathrm{H}$, naphthyl-H), 7.77 (d, $1 \mathrm{H}, J=8.2 \mathrm{~Hz}$, naphthyl- $\mathrm{H}), 7.83(\mathrm{~d}, 1 \mathrm{H}, J=7.6 \mathrm{~Hz}$, naphthyl-H), $7.92(\mathrm{~d}, 1 \mathrm{H}, J=8.0 \mathrm{~Hz}$, naphthyl-H), 8.02 (d, $1 \mathrm{H}, J=8.1 \mathrm{~Hz}$, naphthyl-H), 9.23 (br s, $1 \mathrm{H}, \mathrm{NHCO}$ ), and $15.72(\mathrm{~s}, 1 \mathrm{H}, \mathrm{OH}) ;{ }^{13} \mathrm{C}-\mathrm{NMR}\left(\mathrm{DMSO}-\mathrm{d}_{6}\right) \delta(\mathrm{ppm}) 25.8,26.4$, $110.8,119.8,123.0,124.9,126.9,127.1,127.5,128.3,129.3$, 134.2, 136.9, 165.5, and 198.5 (15C). ESI-MS: $\mathrm{m} / \mathrm{z}, 270.12$ $\left(\mathrm{M}^{+}+\mathrm{H}\right)$. Anal. calcd for $\mathrm{C}_{15} \mathrm{H}_{15} \mathrm{~N}_{3} \mathrm{O}_{2}(269.30)$ : $\mathrm{C}, 66.90 ; \mathrm{H}$, 5.61; and N, 15.60. Found: C, 66.80; H, 5.50; and N, 15.52 .

(3) 2-(Naphthalen-1-yl-diazenyl)-N-tert-butyl-3hydroxybut-2-enamide (6c). Brownish crystalline solid, m.p. $126-127^{\circ} \mathrm{C}$, yield $=59 \%$, and ${ }^{1} \mathrm{H}-\mathrm{NMR}$ (DMSO- $\left.\mathrm{d}_{6}\right)$ $\delta(\mathrm{ppm}) 1.43\left(\mathrm{~s}, 9 \mathrm{H}, \mathrm{C}\left(\mathrm{CH}_{3}\right)_{3}\right), 2.52\left(\mathrm{~s}, 3 \mathrm{H}, \mathrm{C}-\mathrm{CH}_{3}\right)$, 7.63-7.60 (m, 2H, naphthyl-H), 7.74-7.40 (m, 1H, naphthyl- 


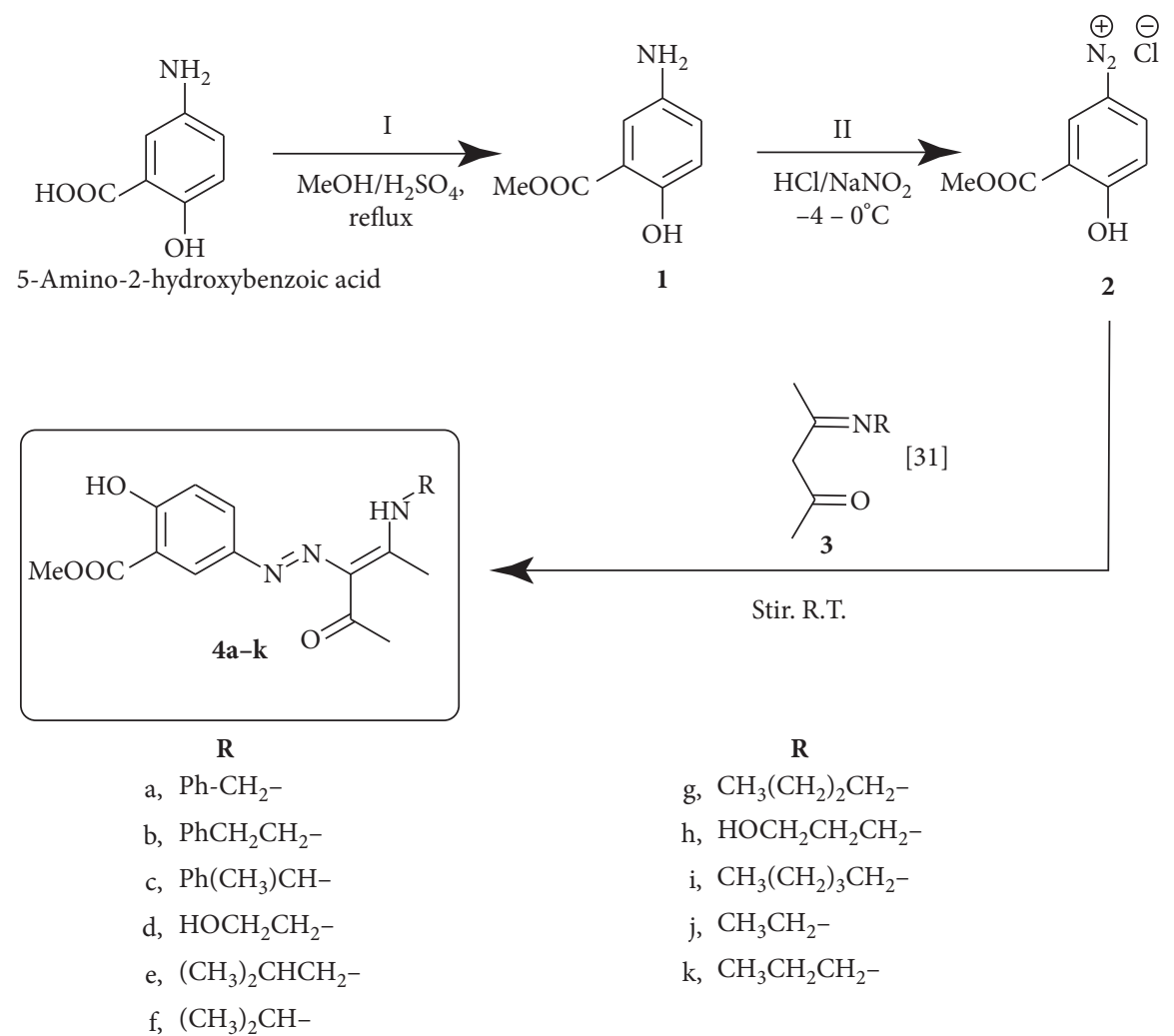

Scheme 1: Synthetic route of compounds $\mathbf{4 a - k}$.

H), 7.78 (d, 1H, $J=8.1 \mathrm{~Hz}$, naphthyl-H), 7.83 (d, 1H, $J=$ $7.6 \mathrm{~Hz}$, naphthyl-H), 7.90 (d, $1 \mathrm{H}, J=8.4 \mathrm{~Hz}$, naphthyl-H), $8.02(\mathrm{~d}, 1 \mathrm{H}, J=8.1 \mathrm{~Hz}$, naphthyl-H), 9.42 (s, 1H, NHCO), and 15.69 (s, 1H, OH); ${ }^{13} \mathrm{C}-\mathrm{NMR}\left(\mathrm{DMSO}-\mathrm{d}_{6}\right) \delta(\mathrm{ppm}) 26.5$, $28.8,51.3,111.0,119.7,123.1,125.1,126.9,127.1,127.6$, $128.4,129.2,134.2,136.9,164.7$, and 199.3 (18C). ESI-MS: $m / z, 312.17\left(\mathrm{M}^{+}+\mathrm{H}\right)$. Anal. calcd for $\mathrm{C}_{18} \mathrm{H}_{21} \mathrm{~N}_{3} \mathrm{O}_{2}$ (311.38): C, 69.43; H, 6.80; and N, 13.49. Found: C, 69.30; H, 6.75; and $\mathrm{N}, 13.40$.

(4) 2-(Naphthalen-1-yl-diazenyl)-3-hydroxy-N-isopropylbut2-enamide (6d). Brownish crystalline solid, m.p. $124-125^{\circ} \mathrm{C}$, yield $=62 \%$, and ${ }^{1} \mathrm{H}-\mathrm{NMR}\left(\mathrm{DMSO}_{-} \mathrm{d}_{6}\right) \delta(\mathrm{ppm}) 1.23(\mathrm{~d}, 6 \mathrm{H}$, $\left.J=6.6 \mathrm{~Hz}, \mathrm{CH}\left(\mathrm{CH}_{3}\right)_{2}\right), 2.52\left(\mathrm{~s}, 3 \mathrm{H}, \mathrm{C}-\mathrm{CH}_{3}\right), 4.11-4.00(\mathrm{~m}$, $\left.1 \mathrm{H}, \mathrm{CH}\left(\mathrm{CH}_{3}\right)_{2}\right), 7.65-7.60(\mathrm{~m}, 2 \mathrm{H}$, naphthyl-H), 7.74-7.70 (m, $1 \mathrm{H}$, naphthyl-H), $7.78(\mathrm{~d}, 1 \mathrm{H}, J=8.1 \mathrm{~Hz}$, naphthyl-H), $7.83(\mathrm{~d}, 1 \mathrm{H}, J=6.8 \mathrm{~Hz}$, naphthyl- $\mathrm{H}), 7.90(\mathrm{~d}, 1 \mathrm{H}, J=8.3 \mathrm{~Hz}$, naphthyl-H), 8.02 (d, $1 \mathrm{H}, J=8.1 \mathrm{~Hz}$, naphthyl-H), 9.26 (br s, $1 \mathrm{H}, \mathrm{NHCO})$, and $15.71(\mathrm{~s}, 1 \mathrm{H}, \mathrm{OH}) ;{ }^{13} \mathrm{C}-\mathrm{NMR}\left(\mathrm{DMSO}-\mathrm{d}_{6}\right)$ $\delta$ (ppm) 22.6, 40.8, 49.1, 110.9, 119.7, 123.1, 125.1, 127.0, $127.1,127.5,128.1,129.3,134.2,136.9,164.2$, and 199.0 (17C). ESI-MS: $m / z, 280.15\left(\mathrm{M}^{+}+\mathrm{H}\right)$. Anal. calcd for $\mathrm{C}_{17} \mathrm{H}_{19} \mathrm{~N}_{3} \mathrm{O}_{2}$ (297.35): C, 68.67; $\mathrm{H}, 6.44$; and $\mathrm{N}, 14.13$. Found: C, 68.60; H, 6.34; and N, 14.00.

(5) 2-(Naphthalen-1-yl-diazenyl)-N-ethyl-3-hydroxybut2-enamide (6e). Brownish crystalline solid, m.p. 130$131^{\circ} \mathrm{C}$, yield $=75 \%$, and ${ }^{1} \mathrm{H}-\mathrm{NMR}\left(\mathrm{DMSO}_{-} \mathrm{d}_{6}\right) \delta(\mathrm{ppm}) 1.17$ $\left(\mathrm{t}, 3 \mathrm{H}, J=7.2 \mathrm{~Hz}, \mathrm{NHCH}_{2} \mathrm{CH}_{3}\right), 2.52\left(\mathrm{~s}, 3 \mathrm{H}, \mathrm{C}-\mathrm{CH}_{3}\right)$, 3.38-3.34 (m, 2H, $\left.\mathrm{NHCH}_{2} \mathrm{CH}_{3}\right), 7.64-7.60(\mathrm{~m}, 2 \mathrm{H}$, naphthyl-H), 7.74-7.70 $(\mathrm{m}, 1 \mathrm{H}$, naphthyl- $\mathrm{H}), 7.77(\mathrm{~d}, 1 \mathrm{H}$, $J=8.1 \mathrm{~Hz}$, naphthyl-H), $7.82(\mathrm{~d}, 1 \mathrm{H}, J=7.6 \mathrm{~Hz}$, naphthyl- $\mathrm{H})$, $7.91(\mathrm{~d}, 1 \mathrm{H}, J=8.4 \mathrm{~Hz}$, naphthyl-H), $8.02(\mathrm{~d}, 1 \mathrm{H}, J=8.1 \mathrm{~Hz}$, naphthyl-H), 9.33 (br s, 1H, NHCO), and 15.72 (s, 1H, OH); ${ }^{13} \mathrm{C}-\mathrm{NMR}$ (DMSO-d $\left.{ }_{6}\right) \delta(\mathrm{ppm}) 14.9,26.5,33.60,110.9$, $119.8,123.0,125.0,126.9,127.1,127.5,128.2,129.3,134.2$, 136.9, 164.8, and 198.7 (16C). ESI-MS: $m / z, 284.14\left(\mathrm{M}^{+}+\mathrm{H}\right)$. Anal. calcd for $\mathrm{C}_{16} \mathrm{H}_{17} \mathrm{~N}_{3} \mathrm{O}_{2}$ (283.33): C, 67.83; $\mathrm{H}, 6.05$; and N, 14.83. Found: C, 67.74; H, 6.00; and N, 14.78 .

(6) 2-(Naphthalen-1-yl-diazenyl)-N-butyl-3-hydroxybut2-enamide (6f). Brownish crystalline solid, m.p. $142-143^{\circ} \mathrm{C}$, yield $=71 \%$, and ${ }^{1} \mathrm{H}-\mathrm{NMR}\left(\mathrm{DMSO}-\mathrm{d}_{6}\right) \delta(\mathrm{ppm}) 0.93(\mathrm{t}, 3 \mathrm{H}$, $\left.J=7.3 \mathrm{~Hz}, \mathrm{NHCH}_{2} \mathrm{CH}_{2} \mathrm{CH}_{2} \mathrm{CH}_{3}\right), 1.38-1.35(\mathrm{~m}, 2 \mathrm{H}, J=$ $\left.7.6 \mathrm{~Hz}, \mathrm{NHCH}_{2} \mathrm{CH}_{2} \mathrm{CH}_{2} \mathrm{CH}_{3}\right), 1.57-1.54(\mathrm{~m}, 2 \mathrm{H}, J=7.3 \mathrm{~Hz}$, $\left.\mathrm{NHCH}_{2} \mathrm{CH}_{2} \mathrm{CH}_{2} \mathrm{CH}_{3}\right) 2.53$ (s, 3H, C-CH $\left.\mathrm{CH}_{3}\right), 3.36$ (q, 2H, J = $7.0 \mathrm{~Hz}, \mathrm{NHCH}_{2} \mathrm{CH}_{2} \mathrm{CH}_{2} \mathrm{CH}_{3}$ ), 7.65-1.62 (m, 2H, naphthyl$\mathrm{H})$, 7.75-7.71 (m, 1H, naphthyl- $\mathrm{H}), 7.77(\mathrm{~d}, 1 \mathrm{H}, J=8.1 \mathrm{~Hz}$, naphthyl-H), $7.82(\mathrm{~d}, 1 \mathrm{H}, J=7.5 \mathrm{~Hz}$, naphthyl-H), 7.91 (d, $1 \mathrm{H}, J=8.4 \mathrm{~Hz}$, naphthyl-H), $8.02(\mathrm{~d}, 1 \mathrm{H}, J=8.1 \mathrm{~Hz}$, naphthyl-H), 9.36 (br s, 1H, NHCO), and 15.72 (s, 1H, OH); ${ }^{13} \mathrm{C}-\mathrm{NMR}$ (DMSO-d 6 ) $\delta(\mathrm{ppm}) 14.1,20.2,26.5,31.3,38.3$, $110.9,119.8,123.1,125.1,126.9,127.1,127.5,128.2,129.3$, 134.2, and 136.9, 164.9, 198.8 (18C). ESI-MS: $m / z, 312.17(\mathrm{M}$ ${ }^{+}+\mathrm{H}$ ). Anal. calcd for $\mathrm{C}_{18} \mathrm{H}_{21} \mathrm{~N}_{3} \mathrm{O}_{2}$ (311.38): C, 69.43; $\mathrm{H}$, 6.80; and N, 13.49. Found: C, 69.35; H, 6.72; and N, 13.40.

(7) 2-(Naphthalen-1-yl-diazenyl)-N-sec-butyl-3-hydroxybut2-enamide (6g). Yellow crystalline solid, m.p. 105-106 ${ }^{\circ} \mathrm{C}$, yield $=55 \%$, and ${ }^{1} \mathrm{H}-\mathrm{NMR}\left(\mathrm{CDCl}_{3}\right) \delta(\mathrm{ppm}) 1.01(\mathrm{t}, 3 \mathrm{H}$, $\left.J=7.4 \mathrm{~Hz}, \mathrm{CH}_{3} \mathrm{CH}_{2} \mathrm{CH}(\mathrm{NH}) \mathrm{CH}_{3}\right), 1.28(\mathrm{~d}, 3 \mathrm{H}, J=6.6 \mathrm{~Hz}$, $\left.\mathrm{CH}_{3} \mathrm{CH}_{2} \mathrm{CH}(\mathrm{NH}) \mathrm{CH}_{3}\right), \quad 1.66-1.60\left(\mathrm{~m}, 2 \mathrm{H}, \mathrm{CH}_{3} \mathrm{CH}_{2} \mathrm{CH}\right.$ $\left.(\mathrm{NH}) \mathrm{CH}_{3}\right), 2.62\left(\mathrm{~s}, 3 \mathrm{H}, \mathrm{C}-\mathrm{CH}_{3}\right), 4.12-4.08(\mathrm{~m}, 1 \mathrm{H}$, $\left.\mathrm{CH}_{3} \mathrm{CH}_{2} \mathrm{CH}(\mathrm{NH}) \mathrm{CH}_{3}\right)$ 7.56-7.52 (m, 3H, naphthyl-H), 


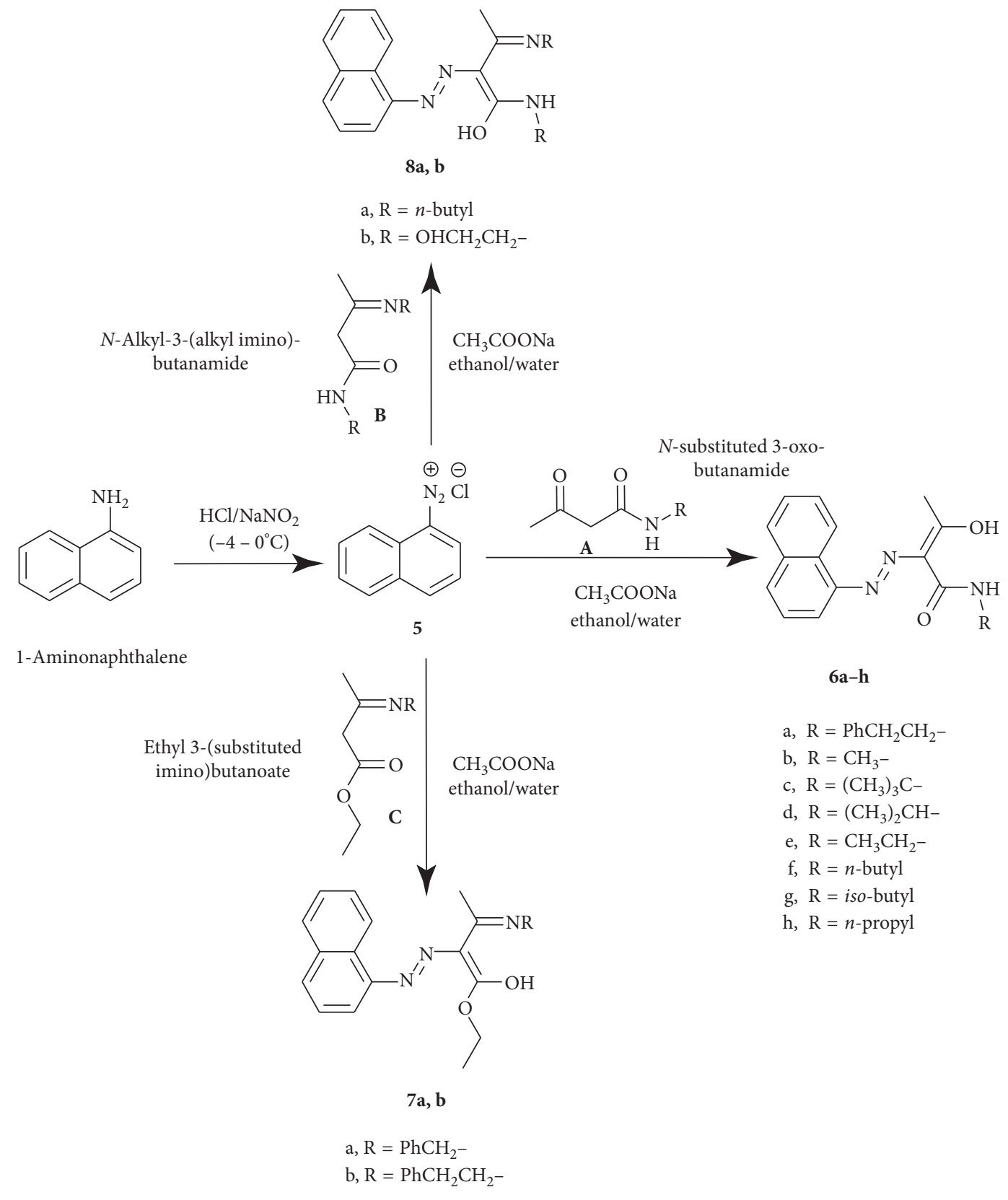

Scheme 2: Synthetic route of compounds 6-8.

$7.68(\mathrm{~d}, 1 \mathrm{H}, J=8.0 \mathrm{~Hz}$, naphthyl-H), $7.82(\mathrm{~d}, 1 \mathrm{H}, J=8.0 \mathrm{~Hz}$, naphthyl-H), $7.90(\mathrm{~d}, 1 \mathrm{H}, J=7.6 \mathrm{~Hz}$ naphthyl-H), 8.07 (d, $1 \mathrm{H}, J=7.9 \mathrm{~Hz}$ naphthyl-H), 9.40 (br s, $1 \mathrm{H}, \mathrm{NHCO}$ ), and $15.81(\mathrm{~s}, 1 \mathrm{H}, \mathrm{OH}) ;{ }^{13} \mathrm{C}-\mathrm{NMR}\left(\mathrm{CDCl}_{3}\right) \delta(\mathrm{ppm}) .10 .4,20.3$, 26.2, 29.4, 46.0, 110.7, 120.2, 123.5, 124.8, 126.1, 126.4, 126.7, 127.6, 128.6, 134.1, 137.2, 164.7, and 199.4 (18C). ESI-MS: $m / z$, $312.16\left(\mathrm{M}^{+}+\mathrm{H}\right)$. Anal. calcd for $\mathrm{C}_{18} \mathrm{~N}_{21} \mathrm{~N}_{3} \mathrm{O}_{2}$ (311.38): C, 69.43; H, 6.80; and N, 13.49. Found: C, 69.35; H, 6.72; and $\mathrm{N}, 13.40$.

(8) 2-(Naphthalen-1-yl-diazenyl)-3-hydroxy-N-propylbut2-enamide (6h). Brownish crystalline solid, m.p. $157-158^{\circ} \mathrm{C}$, yield $=65 \%$, and ${ }^{1} \mathrm{H}-\mathrm{NMR}\left(\mathrm{CDCl}_{3}\right) \delta(\mathrm{ppm}) 1.04(\mathrm{t}, 3 \mathrm{H}, J=$ $\left.7.4 \mathrm{~Hz}, \mathrm{CONHCH}_{2} \mathrm{CH}_{2} \mathrm{CH}_{3}\right), 1.68-1.63(\mathrm{~m}, 2 \mathrm{H}, J=7.3 \mathrm{~Hz}$, $\left.\mathrm{CONHCH}_{2} \mathrm{CH}_{2} \mathrm{CH}_{3}\right), 2.62\left(\mathrm{~s}, 3 \mathrm{H}, \mathrm{C}-\mathrm{CH}_{3}\right), 3.42(\mathrm{q}, 2 \mathrm{H}$, $\left.J=7.0 \mathrm{~Hz}, \quad \mathrm{CONHCH}_{2} \mathrm{CH}_{2} \mathrm{CH}_{3}\right), \quad 7.57-7.53(\mathrm{~m}, 3 \mathrm{H}$,
naphthyl-H), 7.68 (d, $1 \mathrm{H}, J=8.1 \mathrm{~Hz}$, naphthyl-H), 7.82 (d, $1 \mathrm{H}, J=7.5 \mathrm{~Hz}$, naphthyl-H), $7.90(\mathrm{~d}, 1 \mathrm{H}, J=7.5 \mathrm{~Hz}$, naphthyl-H), 8.07 (br d, $1 \mathrm{H}, J=8.5 \mathrm{~Hz}$, naphthyl-H), 9.54 (br s, 1H, NHCO), and 15.79 (s, 1H, OH); ${ }^{13} \mathrm{C}-\mathrm{NMR}$ $\left(\mathrm{CDCl}_{3}\right) \delta(\mathrm{ppm}) \quad 11.6,22.6,26.2,40.4,110.7,120.2$, $123.5,124.9,126.1,126.4,126.7,127.6,128.6,134.1,137.1$, 165.3, and 199.4 (17C). ESI-MS: $m / z, 298.15\left(\mathrm{M}^{+}+\mathrm{H}\right)$. Anal. calcd for $\mathrm{C}_{17} \mathrm{H}_{19} \mathrm{~N}_{3} \mathrm{O}_{2}$ (297.35): C, 68.67; $\mathrm{H}, 6.44$; and $\mathrm{N}$, 14.13. Found: C, 68.55; H, 6.36; and N, 14.04 .

(9) Ethoxy 3-(benzylimino)-2-(naphthalen-1-yl-diazenyl) but-1-en-1-ol (7a). Brownish crystalline solid, m.p. 110$111^{\circ} \mathrm{C}$, yield $=77 \%$, and ${ }^{1} \mathrm{H}-\mathrm{NMR}\left(\mathrm{DMSO}^{\left.-\mathrm{d}_{6}\right)} \delta(\mathrm{ppm}) 1.32\right.$ $\left(\mathrm{t}, 3 \mathrm{H}, J=7.1 \mathrm{~Hz}, \mathrm{OCH}_{2} \mathrm{CH}_{3}\right), 2.59\left(\mathrm{~s}, 3 \mathrm{H}, \mathrm{C}-\mathrm{CH}_{3}\right), 4.27$ (q, $\left.2 \mathrm{H}, J=7.1 \mathrm{~Hz}, \mathrm{OCH}_{2} \mathrm{CH}_{3}\right), 4.83\left(\mathrm{~s}, 2 \mathrm{H}, J=3.4 \mathrm{~Hz}, \mathrm{ArCH}_{2} \mathrm{~N}\right)$, 7.50-4.46 (m, 9H, naphthyl-H and $\mathrm{ArH}), 7.65$ (d, 1H, $J=$ 
TABLE 1: Inhibition percentages of different synthesized compounds against MCF-7, A549, and HT-29 cell line.

\begin{tabular}{|c|c|c|c|}
\hline \multirow{2}{*}{ Compound } & \multicolumn{3}{|c|}{ Inhibition percentage \pm SD (\%) } \\
\hline & MCF-7 & A549 & HT-29 \\
\hline $4 \mathrm{a}$ & $23 \pm 3$ & $34 \pm 4$ & $0.0 \pm 8$ \\
\hline $4 b$ & $4 \pm 5$ & $6 \pm 3$ & $0.0 \pm 8$ \\
\hline $4 c$ & $35 \pm 3$ & $10 \pm 8$ & $0.0 \pm 3$ \\
\hline $4 d$ & $10 \pm 5$ & $9 \pm 8$ & $12 \pm 8$ \\
\hline $4 \mathrm{e}$ & $10 \pm 2$ & $3 \pm 9$ & $0.0 \pm 4$ \\
\hline $4 \mathrm{f}$ & $7 \pm 3$ & $0.0 \pm 8$ & $0.0 \pm 6$ \\
\hline $4 g$ & $13 \pm 7$ & $0.0 \pm 6$ & $13 \pm 7$ \\
\hline $4 \mathrm{~h}$ & $10 \pm 5$ & $11 \pm 5$ & $8 \pm 6$ \\
\hline $4 \mathrm{i}$ & $16 \pm 5$ & $15 \pm 7$ & $13 \pm 4$ \\
\hline $4 \mathrm{j}$ & $11 \pm 4$ & $13 \pm 6$ & $9 \pm 6$ \\
\hline $4 \mathrm{k}$ & $13 \pm 7$ & $10 \pm 5$ & $6 \pm 6$ \\
\hline $6 a$ & $12 \pm 6$ & $7 \pm 4$ & $0.0 \pm 7$ \\
\hline $6 \mathrm{~b}$ & $15 \pm 3$ & $15 \pm 4$ & $6 \pm 7$ \\
\hline $6 c$ & $8 \pm 4$ & $5 \pm 5$ & $0.0 \pm 4$ \\
\hline $6 \mathrm{~d}$ & $11 \pm 5$ & $3 \pm 6$ & $9 \pm 7$ \\
\hline $6 e$ & $6 \pm 5$ & $2 \pm 8$ & $0.0 \pm 7$ \\
\hline $6 f$ & $20 \pm 2$ & $0.0 \pm 7$ & $0.0 \pm 8$ \\
\hline $6 g$ & $1 \pm 6$ & $0.0 \pm 8$ & $0.0 \pm 8$ \\
\hline $6 \mathrm{~h}$ & $10 \pm 5$ & $0.0 \pm 6$ & $2 \pm 8$ \\
\hline $7 a$ & $11 \pm 4$ & $17 \pm 2$ & $11 \pm 6$ \\
\hline $7 b$ & $10 \pm 3$ & $0.0 \pm 6$ & $39 \pm 7$ \\
\hline $8 a$ & $22 \pm 5$ & $0.0 \pm 6$ & $0.0 \pm 8$ \\
\hline $8 b$ & $2 \pm 4$ & $0.0 \pm 3$ & $0.0 \pm 8$ \\
\hline
\end{tabular}

Data are represented as mean \pm SD.

$7.5 \mathrm{~Hz}$, naphthyl-H), $7.72(\mathrm{~d}, 1 \mathrm{H}, J=8.1 \mathrm{~Hz}$, naphthyl-H), $7.88(\mathrm{~d}, 1 \mathrm{H}, J=8.2 \mathrm{~Hz}$, naphthyl-H), and $14.90(\mathrm{~s}, 1 \mathrm{H}, \mathrm{OH})$; ${ }^{13}$ C-NMR (DMSO-d ${ }_{6}$ ) $\delta$ (ppm) 14.9, 17.2, 50.2, 60.3, 111.2, $121.9,124.1,126.1,126.4,126.4,126.6,127.2,128.1,128.5$, $129.3,129.5,134.3,136.9,137.9,166.7$, and 198.7 (23C). ESIMS: $m / z, 374.19\left(\mathrm{M}^{+}+\mathrm{H}\right)$. Anal. calcd for $\mathrm{C}_{23} \mathrm{H}_{23} \mathrm{~N}_{3} \mathrm{O}_{2}$ (373.45): C, 73.97; H, 6.21; and N, 11.25. Found: C, 73.85; H, 6.12; and $\mathrm{N}, 11.20$.

(10) Ethoxy 2-(naphthalen-1-yl-diazenyl)-3(phenethylimino)but-1-en-1-ol (7b). Brownish crystalline solid, m.p. $142-143^{\circ} \mathrm{C}$, yield $=72 \%$, and ${ }^{1} \mathrm{H}-\mathrm{NMR}$ (DMSO$\left.\mathrm{d}_{6}\right) \delta(\mathrm{ppm}) 1.30\left(\mathrm{t}, 3 \mathrm{H}, J=7.1 \mathrm{~Hz}, \mathrm{OCH}_{2} \mathrm{CH}_{3}\right), 2.48(\mathrm{~s}, 3 \mathrm{H}$, C- $\left.\mathrm{CH}_{3}\right), 3.08\left(\mathrm{t}, 2 \mathrm{H}, J=7.1 \mathrm{~Hz}, \mathrm{ArCH}_{2} \mathrm{CH}_{2} \mathrm{~N}\right), 3.89(\mathrm{t}, 2 \mathrm{H}$, $\left.J=6.9 \mathrm{~Hz}, \operatorname{ArCH}_{2} \mathrm{CH}_{2} \mathrm{~N}\right), 4.23(\mathrm{q}, 3 \mathrm{H}, J=7.1 \mathrm{~Hz}$, $\left.\mathrm{OCH}_{2} \mathrm{CH}_{3}\right), 7.20-7.15$ (m, $1 \mathrm{H}$, naphthyl-H), 7.28-7.24 (m, $2 \mathrm{H}$, naphthyl-H), 7.32-7.28 (m, 2H, naphthyl-H), 7.58-7.55 (m, 4H, ArH), 7.78-7.74 (m, 1H, naphthyl-H), 7.96-7.92 (m, $1 \mathrm{H}$, naphthyl-H), and 14.59 (s, 1H, OH); ${ }^{13} \mathrm{C}-\mathrm{NMR}$ (DMSO$\left.\mathrm{d}_{6}\right) \delta$ (ppm). 14.9, 16.5, 35.9, 46.4, 60.1, 111.9, 122.6, 125.1, $126.3,126.5,126.7,126.9,127.5,128.6,128.9,129.2,129.2$, $134.4,136.9,138.9,166.7$, and 198.7 (24C). ESI-MS: $m / z, 388.19$ $\left(\mathrm{M}^{+}+\mathrm{H}\right)$. Anal. calcd for $\mathrm{C}_{24} \mathrm{H}_{25} \mathrm{~N}_{3} \mathrm{O}_{2}$ (387.47): C, 74.39; $\mathrm{H}$, 6.50; and N, 10.84. Found: C, 74.18; H, 6.42; and N, 10.76.

(11) 1-(Butylamino)-3-(butylimino)-2-[(1-(naphthalen-1$y$ l)diazene]but-1-en-1-ol (8a). Brownish crystalline solid, m. p. $91-92^{\circ} \mathrm{C}$, yield $=71 \%$, and ${ }^{1} \mathrm{H}-\mathrm{NMR}\left(\right.$ DMSO-d $\left._{6}\right) \delta(\mathrm{ppm})$. $0.93\left(\mathrm{t}, 3 \mathrm{H}, J=6.4 \mathrm{~Hz}, \mathrm{NHCH}_{2} \mathrm{CH}_{2} \mathrm{CH}_{2} \mathrm{CH}_{3}\right), 0.96(\mathrm{t}, 3 \mathrm{H}$, $\left.J=7.3 \mathrm{~Hz}, \mathrm{NCH}_{2} \mathrm{CH}_{2} \mathrm{CH}_{2} \mathrm{CH}_{3}\right), 1.46-1.40(\mathrm{~m}, 4 \mathrm{H}, J=$ $7.7 \mathrm{~Hz}, \quad \mathrm{NHCH}_{2} \mathrm{CH}_{2} \mathrm{CH}_{2} \mathrm{CH}_{3}$ and $\left.\mathrm{NCH}_{2} \mathrm{CH}_{2} \mathrm{CH}_{2} \mathrm{CH}_{3}\right)$, 1.61 (q, $\left.2 \mathrm{H}, \mathrm{NHCH}_{2} \mathrm{CH}_{2} \mathrm{CH}_{2} \mathrm{CH}_{3}\right), 1.67-1.64(\mathrm{~m}, 2 \mathrm{H}$, $\mathrm{NCH}_{2} \mathrm{CH}_{2} \mathrm{CH}_{2} \mathrm{CH}_{3}$ ), 2.62 (s, 3H, C- $\mathrm{CH}_{3}$ ), 3.39 (q, $2 \mathrm{H}$,
$\left.J=6.4 \mathrm{~Hz}, \mathrm{NHCH}_{2} \mathrm{CH}_{2} \mathrm{CH}_{2} \mathrm{CH}_{3}\right), 3.65(\mathrm{q}, 2 \mathrm{H}, J=6.8 \mathrm{~Hz}$, $\mathrm{NCH}_{2} \mathrm{CH}_{2} \mathrm{CH}_{2} \mathrm{CH}_{3}$ ), 7.56-7.52 (m, 4H, naphthyl-H), 7.76 (d, $1 \mathrm{H}, J=8.2 \mathrm{~Hz}$, naphthyl-H), 7.95-7.90 $(\mathrm{m}, 1 \mathrm{H}$, naphthyl$\mathrm{H}), 8.4(\mathrm{~d}, 1 \mathrm{H}, J=7.4 \mathrm{~Hz}$, naphthyl-H), 11.3 (br s, $1 \mathrm{H}, \mathrm{NH}$ ), and $15.37(\mathrm{~s}, 1 \mathrm{H}, \mathrm{OH}) ;{ }^{13} \mathrm{C}-\mathrm{NMR}\left(\mathrm{DMSO}_{\mathrm{d}}\right) \delta(\mathrm{ppm}) 14.1$, $14.2,14.7,20.0,20.4,31.4,31.7,37.9,44.0,111.3,121.3,122.8$, 126.0, 126.3, 126.8, 126.8, 128.6, 129.0, 134.5, 148.7, 165.9, and $172.7(22 \mathrm{C})$. ESI-MS: $m / z, 367.25\left(\mathrm{M}^{+}+\mathrm{H}\right)$. Anal. calcd for $\mathrm{C}_{22} \mathrm{H}_{30} \mathrm{~N}_{4} \mathrm{O}$ (366.50): C, 72.10; H, 8.25; and $\mathrm{N}, 15.29$. Found: C, 72.00; H, 8.18; and N, 15.20.

(12) N-(2-hydroxyethyl imino)-3-(2-hydroxyethylamino)2-(naphthalen-1-yldiazenyl)but-2-en-2-ol (8b). Brownish crystalline solid, m.p. $157-158^{\circ} \mathrm{C}$, yield $=70 \%$, and ${ }^{1} \mathrm{H}-\mathrm{NMR}$ $\left(\right.$ DMSO-d $\left._{6}\right) \delta(\mathrm{ppm}) 2.64\left(\mathrm{~s}, 3 \mathrm{H}, \mathrm{C}-\mathrm{CH}_{3}\right), 3.47(\mathrm{q}, 3 \mathrm{H}, J=$ $\left.5.3 \mathrm{~Hz}, \mathrm{CONHCH}_{2} \mathrm{CH}_{2} \mathrm{OH}\right), \quad 3.62-3.66(\mathrm{~m}, 6 \mathrm{H}, \mathrm{CON}-$ $\mathrm{HCH}_{2} \mathrm{CH}_{2} \mathrm{OH}$ and $\mathrm{CNCH}_{2} \mathrm{CH}_{2} \mathrm{OH}$ ), 4.95 (t, $1 \mathrm{H}, J=4.6 \mathrm{~Hz}$, $\left.\mathrm{CONHCH}_{2} \mathrm{CH}_{2} \mathrm{OH}\right), 5.08\left(\mathrm{t}, 1 \mathrm{H}, J=4.4 \mathrm{~Hz}, \mathrm{CNCH}_{2} \mathrm{CH}_{2} \mathrm{OH}\right)$, 7.54-7.50 (m, 4H, naphthyl-H), $7.74(\mathrm{~d}, 1 \mathrm{H}, J=7.8 \mathrm{~Hz}$, naphthyl-H), 7.94-7.88 (m, $1 \mathrm{H}$, naphthyl-H), 8.74-8.70 (m, $1 \mathrm{H}$, naphthyl-H), 11.40 (br s, $1 \mathrm{H}, \mathrm{NH})$, and $15.37(\mathrm{~s}, 1 \mathrm{H}, \mathrm{OH}$ ); ${ }^{13}$ C-NMR (DMSO-d $\mathrm{d}_{6}$ ) $\delta$ (ppm).15.0, 41.1, 47.1, 60.0, 60.5, $111.1,121.3,123.9,126.3,126.4,126.7,126.8,128.2,129.2$, 134.5, 148.8, 165.8, and 172.8 (18C). ESI-MS: $m / z, 343.18\left(\mathrm{M}^{+}\right.$ $+\mathrm{H})$. Anal. calcd for $\mathrm{C}_{18} \mathrm{H}_{22} \mathrm{~N}_{4} \mathrm{O}_{3}$ (342.39): C, 63.14; $\mathrm{H}, 6.48$; and N, 16.36. Found: C, 63.00; H, 6.40; and N, 16.30 .

2.2. Cytotoxicity Assay. Three cancer cell lines were used in the current study: MCF-7 (human breast adenocarcinoma), A549 (lung carcinoma), and HT-29 (human colorectal adenocarcinoma). Cancer cell lines were obtained from SigmaAldrich Chemical Company, USA, and were seeded in Dulbecco's Modified Eagle Media (DMEM) supplemented with $10 \%$ heat-inactivated fetal bovine serum (FBS), $100 \mathrm{IU} / \mathrm{mL}$ penicillin, $100 \mathrm{mg} / \mathrm{mL}$ streptomycin, and $2 \mathrm{mM}$ glutamine. The cells were maintained in a humidified atmosphere with $5 \% \mathrm{CO}_{2}$ at $37^{\circ} \mathrm{C}$.

2.2.1. Antiproliferative Activity. The antiproliferative activity of the tested compounds was measured using the 3-(4,5dimethylthiazol-2-yl)-2,5-diphenyltetrazolium bromide (MTT) assay as mentioned before [28-30]. In brief, cells were seeded in 96 -well plates $24 \mathrm{~h}$ before different treatments. Test compounds were dissolved in dimethyl sulfoxide (DMSO) and further diluted with the cultivation medium. All target compounds (1-23) were preliminary evaluated in vitro at the single concentration of $5 \mu \mathrm{M}$ against different cancer cell lines. Doxorubicin was used in parallel as a positive control drug at the single concentration of $2 \mu \mathrm{M}$. Different tested samples were added to the wells, and the cells were incubated at $37^{\circ} \mathrm{C}$ in a $5 \% \mathrm{CO}_{2}$-humidified incubator for $48 \mathrm{~h}$. After treatment, $10 \mu \mathrm{L}$ of the MTT solution $(5 \mathrm{mg} / \mathrm{mL})$ was added to each well, and the plates were further incubated for $2 \mathrm{~h}$. The media along with the MTT solution was removed and replaced by $100 \mu \mathrm{L}$ of the DMSO solution to dissolve the precipitating formazan crystals. The intensity of the developed color was measured at $570 \mathrm{~nm}$ with an automatic multiwell plate reader Varioskan $^{\mathrm{TM}}$ LUX multimode microplate reader, Thermo Fisher Scientific, 


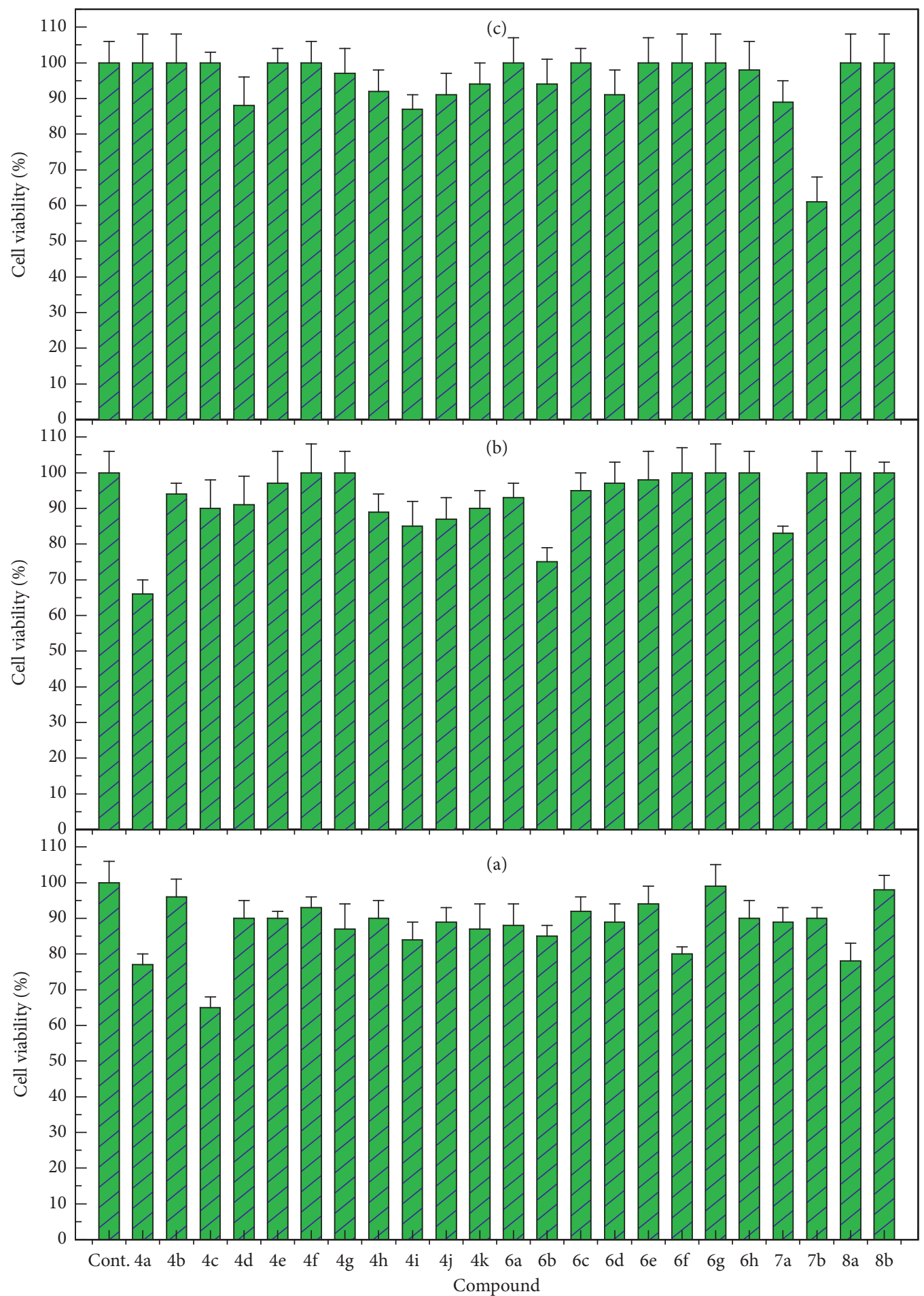

FIGURE 1: Effect of different compounds on the viability of MCF-7, A549 and HT-29 cell line. Data are represented as mean \pm SD. (a) MCF-7; (b) A549; (c) HT-29.

USA). Percentage inhibition of proliferation was calculated as a fraction of the vehicle control.

\section{Results and Discussion}

3.1. Chemistry. In the present work, a series of methyl 2-hydroxy-5-4-oxo-2-(substituted amino)pent-2-en-3-yl) diazenyl)benzoate $4 \mathbf{a}-\mathbf{k}$ were synthesized via the diazotization of methyl 5-amino-2-hydroxybenzoate $\mathbf{2}$ as the starting material. Nitrosation of methyl 5-amino-2hydroxybenzoate $\mathbf{1}$ by using $\mathrm{NaNO}_{2}$ in the presence of $\mathrm{HCl}$ afforded the corresponding diazonium salt derivative $\mathbf{2}$. Treatment of 2 with 4-(substituted amino)pent-3-en-2-one derivatives 3 [31] in absolute ethanol with stirring at room 
temperature gave the corresponding methyl 2-hydroxy5-(4-oxo-2-(substituted amino)pent-2-en-3-yl)diazenyl) benzoate $4 \mathbf{a}-\mathbf{k}$, respectively (Scheme 1).

Additionally, nitrosation of 1-aminonaphthalene by using $\mathrm{NaNO}_{2}$ in the presence of $\mathrm{HCl}$ afforded the corresponding diazonium salt of naphthalen-1-amine 5. Treatment of diazonium compound $\mathbf{5}$ with $\mathrm{N}$-substituted 3-oxobutanamides (A) or ethyl 3-(substituted imino)butanoates (B) gave the corresponding azocompounds $\mathbf{6 a - h}$ and $7 \mathbf{a}, \mathbf{b}$, respectively. Finally, compound 5 was reacted with $\mathrm{N}$-alkyl3-(alkyl imino)butanamides (C) in the presence of sodium acetate to afford the corresponding compounds $\mathbf{8 a}, \mathbf{b}$, respectively (Scheme 2).

3.2. Anticancer Activity. The results of the antiproliferative activity of the synthesized compounds (Table 1, Figure 1) clearly showed that the synthesized compounds greatly varied in their effects on the tested cell lines. All the evaluated 23 compounds showed variable effects on MCF-7 cells. On the contrary, compounds 4f, $\mathbf{6 f}, \mathbf{6 g}, \mathbf{8 a}$, and $\mathbf{8 b}$ were not effective against A549 and HT-29 cells. For MCF-7 cells, the most effective compounds are $\mathbf{4 c}, \mathbf{4 a}$, and $\mathbf{8 a}$, whereas their toxicities were moderate on cells and reached 35,23 , and $22 \%$, respectively. Compounds $6 \mathbf{f}, 4 \mathbf{i}, \mathbf{6 b}, \mathbf{4 k}, \mathbf{6 a}, 4 \mathbf{j}, \mathbf{6 d}$, and 7 a showed lower anticancer activities, and their toxicities obtained at the tested dose recorded $20,16,15,13,12,11,11$, and $11 \%$, respectively. The rest of the synthesized compounds showed weak anticancer activities, where they maximally reduced cell viabilities by $10 \%$. Concerning A549 cells, compounds $\mathbf{4 a}$ and $\mathbf{6} \mathbf{b}$ showed a maximal potent effect, and their percentages of inhibition of cancer cells were obtained as 34 and 25\%, respectively. On the contrary, compounds $\mathbf{7 a}, \mathbf{4 i}, \mathbf{4 j}$, and $\mathbf{4 h}$ showed lower anticancer activities, and their maximal cytotoxicities reached 17,15 , 13 , and $11 \%$, respectively. For HT-29 cells, it can be seen that the most potent compound (compound $7 \mathbf{b}$ ) against HT-29 cells reduced cell growth by about $39 \%$. On the contrary, compounds 4f, 4d, and 7a showed weaker cytotoxic activities ranging only from 13 to $11 \%$. It can be generally concluded that the most potent synthesized compounds are 7b against HT-29 cells, compound 4 a against A549 cells, and compound $4 \mathrm{c}$ against MCF-7 cells.

The abovementioned results showed that different cell lines varied greatly in their response against different synthesized compounds. This correlates well with previously reported results $[32,33]$, where this can be attributed to the inherent differences between different cells in their specific membrane structure and organization. For the synthesized derivatives, our results are in good agreement with those previously reported by Jakopec et al. [34], who reported on the cytotoxic activities of diazenes on several tumor cell lines. They attributed their results to both (1) the attachment of unsubstituted benzene on the amide nitrogen and (2) the extent of compound basicity. Moreover, the length of the conjugation plays a crucial role in the activity of the synthesized derivatives [34].

Generally, it can be observed that the obtained anticancer activities of the newly synthesized diazene candidates are not promising as expected; therefore, the future work is planned to investigate further structural modifications of the prepared derivatives.

\section{Data Availability}

All data used to support the findings of this study are included within the article.

\section{Conflicts of Interest}

The authors declare that they have no conflicts of interest.

\section{Acknowledgments}

The authors are grateful to the Deanship of Scientific Research, King Saud University, for funding this work through research group project "RGP-1435-047."

\section{References}

[1] N. M. Khalifa, M. A. Al-Omar, A. E. Amr, and M. E. Haiba, "HIV-1 and HSV-1 virus activities of some new polycyclic nucleoside pyrene candidates," International Journal of Biological Macromoleculesd, vol. 54, pp. 51-56, 2013.

[2] A. E. Amr, N. A. Abdel-Latif, and M. M. Abdalla, "Synthesis of some new testosterone derivatives fused with substituted pyrazoline ring as $5 \alpha$-reductase inhibitors," Acta Pharmaceutica (Zagreb, Croatia), vol. 56, no. 2, pp. 203-218, 2006.

[3] B. F. Abdel Wahab, S. F. Mohamed, A. E. Amr, and M. M. Abdalla, "Synthesis and reactions of some newly synthesized thiosemicarbazides, triazoles and Schiff bases as antihypertensive $\alpha$-blocking agents," Monatshefte für Chemie - Chemical Monthly, vol. 139, no. 9, pp. 1083-1090, 2008.

[4] N. O. Al-Harbi, S. A. Bahashwan, A. A. Fayed, M. S. Aboonq, and A. E. Amr, "Anti-parkinsonism, hypoglycemic and antimicrobial activities of some new poly ring heterocyclic candidates," International Journal of Biological Macromolecules, vol. 57, pp. 165-173, 2013.

[5] M. M. Abdalla, M. A. Al-Omar, R. A. Al-Salahi, A. E. Amr, and N. M. Sabry, "A new investigation for some steroidal derivatives as anti-alzheimer agents," International Journal of Biological Macromolecules, vol. 51, no. 2, pp. 56-63, 2012.

[6] L. Yurttaş, Y. Özkay, H. K. Gençer, and U. Acar, "Synthesis of some new thiazole derivatives and their biological activity evaluation," Journal of Chemistry, vol. 2015, Article ID 464379, 7 pages, 2015.

[7] K. A. Khan, H. M. Faidallah, and A. M. Asiri, "Synthesis and biological evaluation of new N,N'-Bis(1-substitutedethylidene)-ethane1,2-diamine and their acetyl and trifluoroacetyl derivatives as cytotoxic and antimicrobial agents," Journal of Chemistry, vol. 2013, Article ID 478635, 9 pages, 2013.

[8] Z. A. Kaplancıklı, M. D. Altıntop, B. Sever, Z. Cantürk, and A. Özdemir, "Synthesis and in vitro evaluation of new thiosemicarbazone derivatives as potential antimicrobial agents," Journal of Chemistry, vol. 2016, Article ID 1692540, 7 pages, 2016.

[9] A. E. Amr and M. M. Abdulla, "Synthesis and antiinflammatory activities of new cynopyrane derivatives fused with steroidal nuclei," Archiv der Pharmazie, vol. 339, no. 2, pp. 88-95, 2006. 
[10] L. Pan, N. Hang, C. Zhang et al., "Synthesis and biological evaluation of novel benzimidazole derivatives and analogs targeting the NLRP3 inflammasome," Molecules, vol. 22, no. 2, p. 213, 2017.

[11] C. Holohan, S. Van Schaeybroeck, D. B. Longley, and P. G. Johnston, "Cancer drug resistance: an evolving paradigm," Nature Reviews Cancer, vol. 13, no. 10, pp. 714-726, 2013.

[12] D. M. Marmion, Hand Book of Colorant, Wiley, Hoboken, NJ, USA, 1999.

[13] S. Piotto, S. Concilio, L. Sessa et al., "Synthesis and antimicrobial studies of new antibacterial azo-compounds active against Staphylococcus aureus and Listeria monocytogenes," Molecules, vol. 22, p. 1372, 2017.

[14] S. Concilio, P. Iannelli, L. Sessa et al., "Biodegradable antimicrobial films based on poly (lactic acid) matrices and active azo compounds," Journal of Applied Polymer Science, vol. 132, no. 33, article 42357, 2015.

[15] S. Piotto, S. Concilio, L. Sessa et al., "Novel antimicrobial polymer films active against bacteria and fungi," Polymer Composites, vol. 34, no. 9, pp. 1489-1492, 2013.

[16] S. Piotto, S. Concilio, L. Sessa et al., "Small azobenzene derivatives active against bacteria and fungi," European Journal of Medicinal Chemistry, vol. 68, pp. 178-184, 2013.

[17] L. Sessa, S. Concilio, P. Iannelli, F. De Santis, A. Porta, and S. Piotto, "Antimicrobial azobenzene compounds and their potential use in biomaterials," in Proceedings of the AIP Conference, pp. 16-19, Oudeniz, Turkey, April 2015.

[18] I. M. Awad, A. A. Aly, A. M. Abdel Alim, R. A. Abdel, and S. H. Ahmed, "Synthesis of some 5-azo(4'-substituted benzene-sulphamoyl)-8-hydroxyquinolines with antidotal and antibacterial activities," Journal of Inorganic Biochemistry, vol. 33, no. 2, pp. 77-89, 1998.

[19] S. A. Ibrahim, M. A. Gahami, Z. A. Khafagi, and S. A. Gyar, "Structure and antimicrobial activity of some new azopyrazolone chelates of $\mathrm{Ni}(\mathrm{II})$ and $\mathrm{Cu}(\mathrm{II})$ acetates, sulfates, and nitrates," Journal of Inorganic Biochemistry, vol. 43, no. 1, pp. 1-7, 1991.

[20] A. A. Jarahpour, M. Motamedifar, K. Pakshir, N. Hadi, and Z. Zarei, "Synthesis of novel azo Schiff bases and their antibacterial and antifungal activities," Molecules, vol. 9, no. 10, pp. 815-824, 2004

[21] K. M. Rathod and N. S. Thakre, "Synthesis and antimicrobial activity of azo compounds containing m-cresol moiety," Chemical Science Transactions, vol. 2, no. 1, pp. 25-28, 2013.

[22] S. Samadhiya and H. Halve, "Synthetic utility of schiff bases as potential herbicidal agents," Oriental Journal of Chemistry, vol. 17, pp. 119-122, 2001.

[23] P. Mao, Z. Liu, M. Xie et al., "Naturally occurring methyl salicylate glycosides," Mini-Reviews in Medicinal Chemistry, vol. 14, no. 1, pp. 56-63, 2014.

[24] W. Xin, C. Huang, X. Zhang et al., "Methyl salicylate lactoside inhibits inflammatory response of fibroblast-like synoviocytes and joint destruction in collagen-induced arthritis in mice," British Journal of Pharmacology, vol. 171, no. 14, pp. 35263538, 2014.

[25] X. Zhang, J. Sun, W. Xin et al., “Anti-inflammation effect of methyl salicylate 2 -O- $\beta$-D-lactoside on adjuvant inducedarthritis rats and lipopolysaccharide (LPS)-treated murine macrophages RAW264.7 cells," International Immunopharmacology, vol. 25, no. 1, pp. 88-95, 2015.

[26] A. L. Walpole and M. H. C. Williams, "Aromatic amines as carcinogens in industry," British Medical Bulletin, vol. 14, no. 2, pp. 141-145, 1958.
[27] R. Sirgamalla, A. Kommakula, S. Banoth et al., "Synthesis of amides from aliphatic acids and amines by using of I2/TBHP at room temperature," Chemistry Select, vol. 3, no. 4, pp. 1062-1065, 2018.

[28] H. A. Omar, S. A. Arafa, I. A. Maghrabi, and J. R. Weng, "Sensitization of hepatocellular carcinoma cells to Apo2L/ TRAIL by a novel Akt/NF-kappaB signalling inhibitor," Basic \& Clinical Pharmacology \& Toxicology, vol. 114, no. 6, pp. 464-471, 2014.

[29] H. A. Omar, W. R. Mohamed, S. A. Arafa et al., "Hesperidin alleviates cisplatininduced hepatotoxicity in rats without inhibiting its antitumor activity," Pharmacological Reports, vol. 68 , no. 2, pp. 349-356, 2016.

[30] J. J. Wu, H. A. Omar, Y. R. Lee et al., "6-Shogaol induces cell cycle arrest and apoptosis in human hepatoma cells through pleiotropic mechanisms," European Journal of Pharmacology, vol. 762, pp. 449-458, 2015.

[31] A. Dib, "Synthesis and characterization of schiff bases derived from acetylacetone and their theoretical study," International Journal of Chem Tech Research, vol. 5, no. 1, pp. 204-211, 2013.

[32] E. A. Elsayed, M. A. Sharaf-Eldin, and M. Wadaan, "In vitro evaluation of cytotoxic activities of essential oil from Moringa oleifera seeds on HeLa, HepG2, MCF-7, CACO-2 and L929 cell lines," Asian Pacific Journal of Cancer Prevention, vol. 16, no. 11, pp. 4671-4675, 2015.

[33] E. A. Elsayed, M. Farooq, D. Dailin et al., "In vitro and in vivo biological screening of kefiran polysaccharide produced by Lactobacillus kefiranofaciens," Biomedical Research, vol. 28, no. 2, pp. 594-600, 2017.

[34] S. Jakopec, K. Dubravcic, A. Brozovic, S. Polanc, and M. Osmak, "Structurally similar diazenes exhibit significantly different biological activity," Cell Biology and Toxicology, vol. 22, no. 1, pp. 61-71, 2006. 

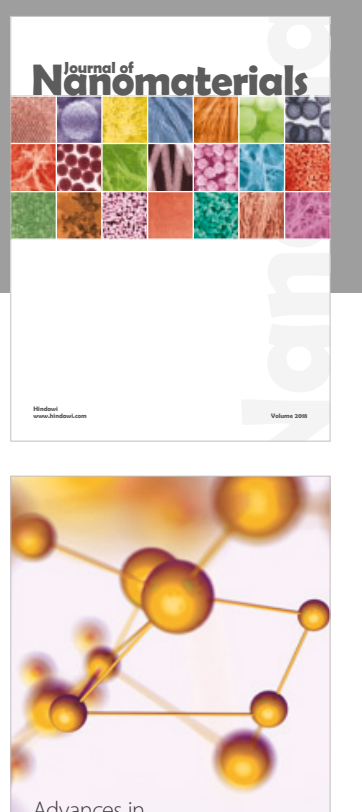

Physical Chemistry
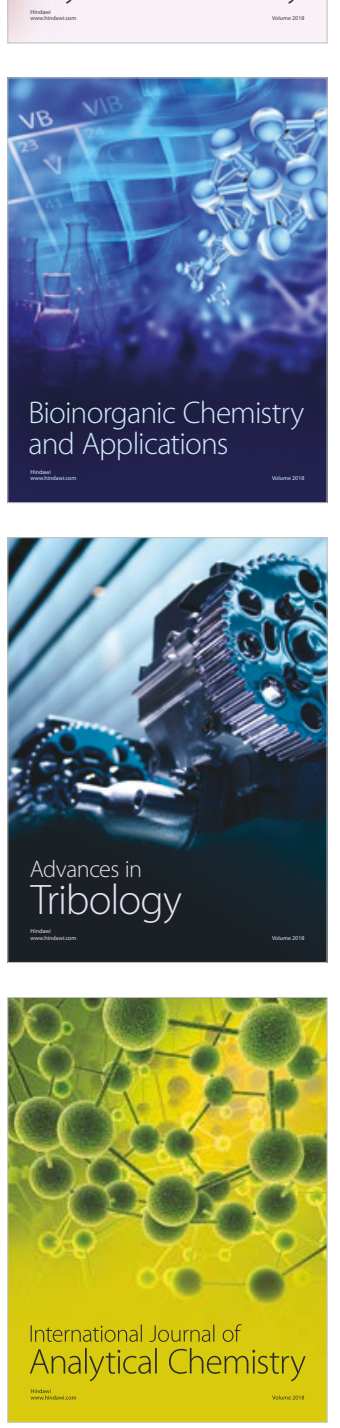

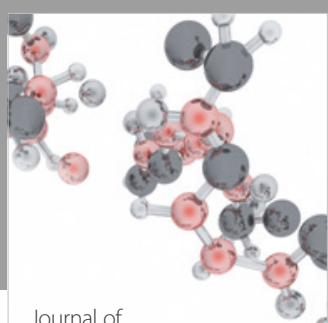

Analytical Methods

in Chemistry

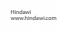

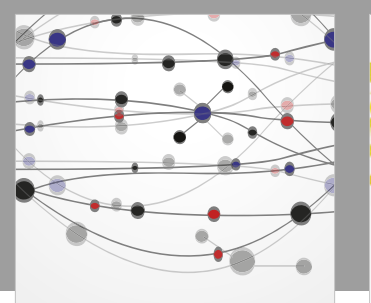

The Scientific World Journal

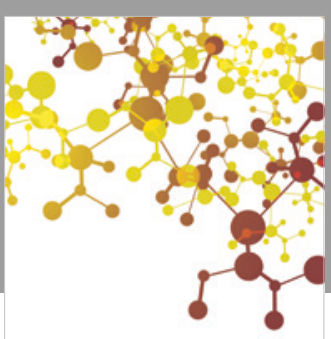

Journal of

Applied Chemistry
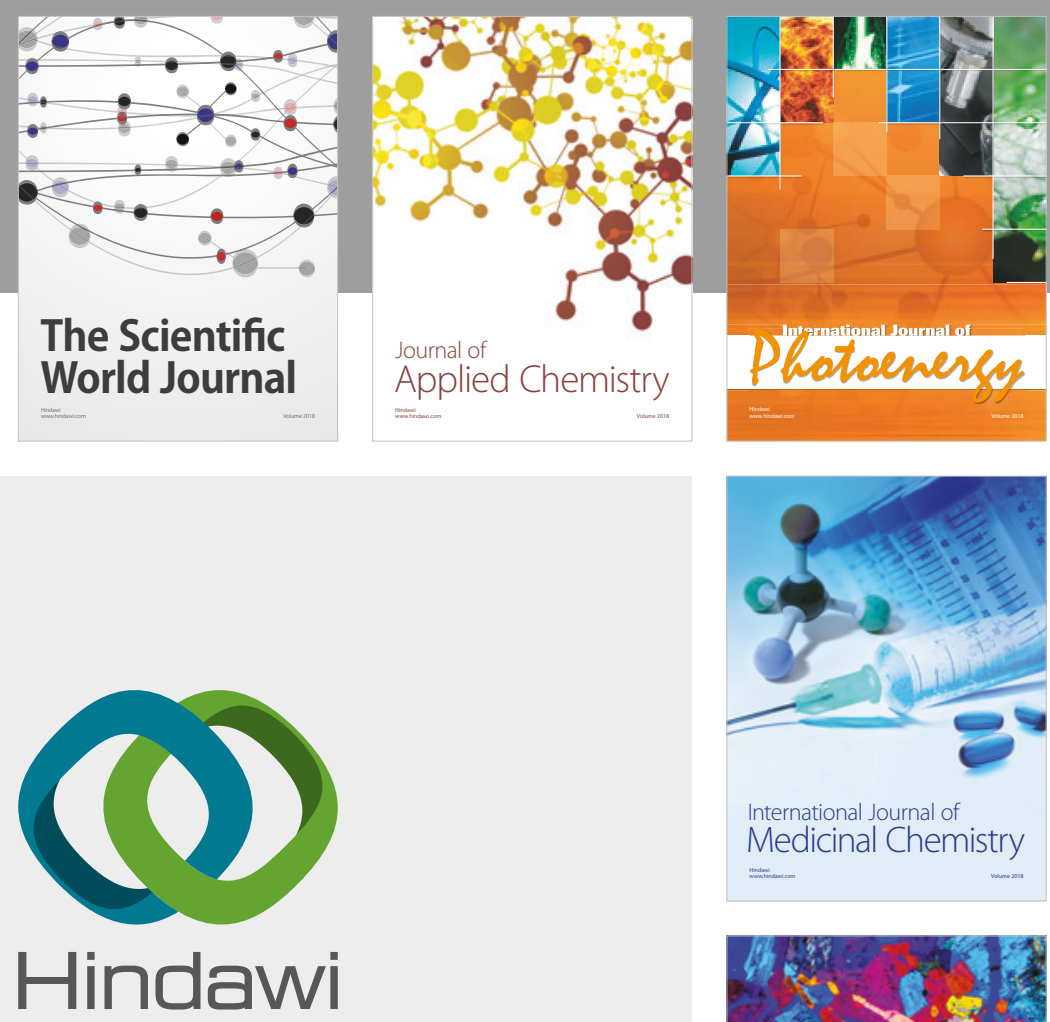

Submit your manuscripts at

www.hindawi.com
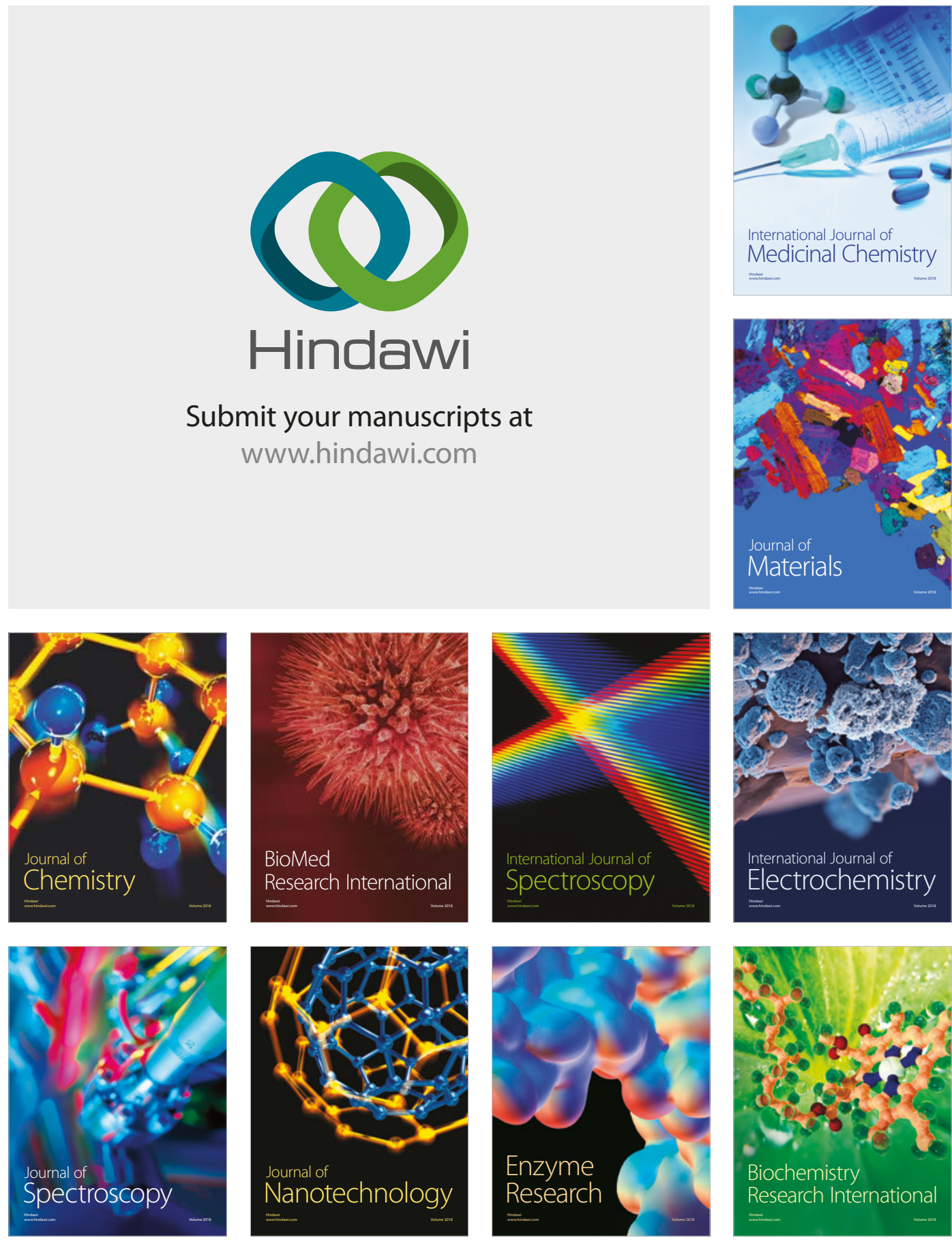
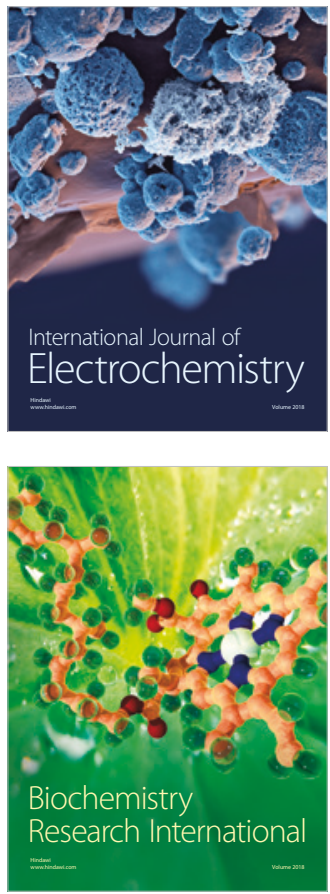